\title{
Le problème des affouillements à l'aval des barrages
}

\section{Scour downstream of dams}

\author{
PAR B. FAUCONNIER \\ INGÉNIEUR A ŔLECTRICIT́ DE FRANCE - R.E.H. ALPES HI
}

Communication présentée à la Société Hydrotechnique de France

(Paris, 27-28 juin 1957)

\begin{abstract}
Pour limiter les afjouillements à l'abul d'un barrage, la méthode la plus satisfaisante consiste a dissiper l'energie des eaux restitues par formation d'un ressaut ordinaire sur un radier de réception.

Une méthode de calcul est proposée, pour déterminer la solution la plas simple d'an radier horizontal. Par itude sur modele, on put encore, sous certaines conditions, trouber des, dispositions qui améliorent sensiblement l'efficacité de lonvragie.

Les essais systematiques effectues a loccasion du barrage de Cadarache ont porte sur les factents suivants :

- profil en long et rugosité de louvrage,

forme des piles,

- nature et mise en ounre des enrochements déversés ì l'aval,

- consignes de manallore,

et ont mis en évidence les principes suivant lesquels il convient de les choisir.
\end{abstract}

L'un des problèmes posés par la construction d'un barrage en rivière est celui de l'évacuation des eaux restituées sans effet destructeur à l'aval. L'énergie correspondant à la surélévation de la ligne piézométrique naturelle, créée artificiellement au droit du barrage, se dissipe à l'aval immédiat de louvrage en provoquant des affouillements dont l'importance peut mettre en danger sa stabilité, ou du moins conduire à une augmentation importante de son cout. Or, on sait que les caractéristiques de l'ouvrage de restitution peuvent faire varier l'importance du déchaussement pour un même débit unitaire.

Une littérature technique très abondante traite de la question et, comme il arrive souvent dans de tels domaines, le projeteur a de la peine à en tirer au moins une idée directrice pour la résolution du cas concret qui se présente à lui. Finalement, il s'adresse en général à la technique du modèle réduit, qu'il s'agisse de constructions nouvelles ou de travaux de réfection (ce dernier cas se présentant d'ailleur's de plus en plus sou-

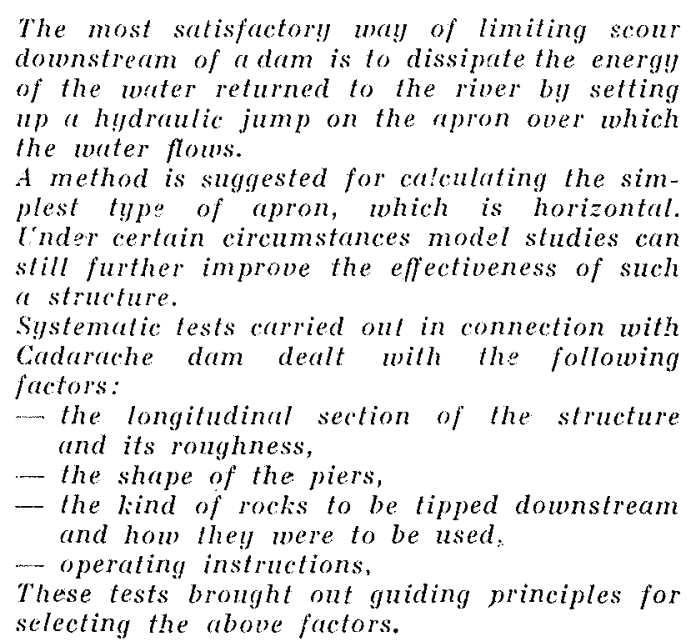

vent). La conclusion de l'étude particulière ainsi engagée, évidemment réduite en délai et en prix, constitue un nouvel apport au problème général des affouillements, sans pour cela faciliter beaucoup la tâche du projeteur suivant.

La présente communication rend compte d'une étude de ce genre, entreprise par l'Electricité de France a l'occasion du barrage de Cadarache sur la Durance, barrage constitué par cinq passes de $25 \mathrm{~m}$ de largeur, équipées de vannes secteurs. Les essais ont été effectués dans un laboratoire situé près d'Aix-en-Provence et construit sous l'égide dụ Laboratoire National d'Frydraulique de Chatou. Nous pensons que l'importance de cette étude (près d'un millier d'heures d'essais) et l'esprit dans lequel on s'est efforcé de la conduire, justifient l'espoir d'une contribution au problème des affouillements, qui dépasse le cadre du cas particulier traité.

Pour dissiper l'énergie cinétique de l'eau à l'aval d'un barrage, il faut d'abord prendre parti entre deux solutions: 
- soit admettre cette dissipation dans le cours d'eau lui-même,

- soit l'assurer par formation d'un ressaut sur un ouvrage spécial de réception (souvent appelé bassin de tranquillisation).

L'expérience a montré que le rejet à l'aval de la lame d'eau est une solution de pis-aller qui présente de sérieux dangers même sur fond rocheux [1] [2]. Le rocher est toujours plus ou moins fissuré et la veine liquide finit à la longue par en provoquer la désagrégation par suite de la répétition des variations de pression et des chocs directs des masses rocheuses déjà détachées.

C'est pourquoi on n'a recours à la première solution que lorsque la deuxième n'est pas possible, ou, du moins, trop coûteuse par rapport au risque couru. C'est surtout le cas des barrages de grande hauteur. Mais il faut remarquer qu'il s'agit alors, en général, d'évacuateurs de crues de barrages réservoirs. Les dispositions à prendre n'ont done à répondre qu'aux conditions posées par des crues de faible fréquence et de courte durée.

Leur principe consiste à rejeter la veine liquide aussi loin que possible du pied de l'ouvrage par incurvation du coursier du déversoir et, en outre, à dissiper le maximum de son énergie. A cet effet, des blocs dissipateurs sont souvent prévus sur le radier même, bien qu'ils $y$ " subissent des efforts très élevés, dus aux vitesses de déversement. L'extrémité du radier est tout particulièrement étudiée sur modèle réduit, afin d'obtenir selon les conditions aval, soit une large dispersion de la veine dans l'air, soit la formation d'un rouleau de fond tendant à remblayer le pied de l'ouvrage (dents de Rehbock). La dissipation complete de l'énergie se faisant dans la masse d'eau aval, on peut mème être obligé de prétablir dans le lit une fosse consolidée spécialement.

Nous nous attacherons, dans ce qui suit, à la deuxième méthode de dissipation de l'énergie par formation d'un ressaut hydraulique. Après un rappel sur les données du problème, nous développerons les procédés d'étude, soit par voie théorique, soit par voie expérimentale, en insistant sur les précautions qu'il est alors indispensable de prendre. Dans le dernier chapitre, nous décrirons la solution à laquelle nous ont conduit les essais sur le barrage de Cadarache.

\section{A) DONNEES DE L'ÉTUdE}

Parmi les données nécessaires à une résolution rationnelle du probleme, nous insisterons seulement sur deux éléments importants souvent négligés.

Il s'agit de la courbe hauteur-débit naturelle et du charriage à travers le barrage.

\section{1" Détermination DE la Covrbe naturelre haU- TEUR-DÉBIT :}

Le niveau naturel aval doit constituer le niveau aval du ressaut que l'on veut former pour dissiper l'énergie. Il est donc essentiel de le connaître avec le maximum de précision. On cherche généralement à l'obtenir en observant une échelle à l'emplacement du barrage et en exploitant une station de jaugeage dans une section rétrécie du lit majeur à proximité.

Or, dans un lit à fond mobile, les variations locales peuvent être très importantes tant en plan (il faut alors déplacer l'échelle après chaque crue) qu'en altitude (à la station de jaugeage du pont de Mirabeau, sur la Durance, les variations de débit sont de $\pm 50 \%$ pour une même hauteur à l'échelle).

C'est pourquoi, pour parvenir à une courbe
$h(\mathrm{Q})$ qui puisse vraiment caractériser une section déterminée de la rivière, il faudrait une durée d'observation très longue, dont on ne dispose malheureusement pas souvent au moment de l'établissement du projet. On peut cependant y suppléer au moyen de la méthode pratique suivante.

Il suffit de relever systématiquement la ligne d'eau d'un troncon de quelques kilomètres de part et d'autre de la section étudiée en ayant préalablement nivelé des points fixes sur les berges. Pour établir le profil en long, on projette chaque point d'eau sur l'axe du lit majeur, et, de la liģne brisée obtenue, on déduit par la méthode des moindres carrés une ligne d'eau moyenne, qui peut être très précise si le nombre de points observés a été suffisant (1) : identité pour un même débit et parallélisme pour des débits différents.

Pour l'extrapolation aux débits exceptionnels, il est possible d'ajuster à la courbe $h(Q)$ obtenue, à partir de ce réseau, une loi de la forme : $Q=a J^{1 / 2} h^{\alpha}$, a et a caractérisant la forme

(1) Au moins un point tous les $500 \mathrm{~m}$. 
et la rugosité de la section transversale de la rivière.

$2^{\circ}$ EFFET DU Charriage A TRAVERS LE barRage SUR LE PHÉNOMÈNE D'AFFOULLEMENT AVAL.

L'évolution de la fosse d'affouillement est très différente selon qu'il peut se produire ou non un passage de gravier à travers le barrage. Dans l'affirmative, l'apport de matériaux qui en résulte dans la fosse ralentit son érosion, la stabilise, ou même en provoque le remblaiement. Si aucun charriage ne se produit, l'évolution de la fosse se présente au contraire dans des conditions de plus en plus défavorables. En effet, le troncon de rivière situé à l'aval subit alors une érosion générale atteignant son maximum à proximité du barrage et réduisant ainsi la stabilité du ressaut sur le radier.

Le problème des affouillements à l'aval d'un barrage est donc lié à celui de l'engravement de la retenue. Des études approfondies, qui sortiraient du cadre de cette note, ont été entreprises sur cette question à l'occasion du barrage de Cadarache.

Il en ressort qu'à partir de la mise en eau et pratiquement jusqu'au moment où le banc d'engravement parvient à proximité de l'ouvrage, le charriage vers l'aval est nul, ou commence seulement à partir d'un débit élevé (crue millénaire pour le barrage de Cadarache). Selon l'importance de la retenue, la durée de celte première phase d'évolution peut ètre très longue (à Cada- rache, elle a pu être évaluée à environ 25 ans sans qu'il ait été tenu compte de la réduction du charriage naturel du fait des aménagements projetés à l'amont).

Par la suite, le charriage à travers le barrage s'accroît, mais de facon irrégulière, car il dépend notamment du tracé du lit mineur amont et du dispositif de dégravement de la prise d'eau. De toute facon, il reste longtemps inférieur au charriage naturel de la rivière qui parvient à l'amont de la retenue et se répartit très irrégulièrement entre les passes, même si le débit liquide était également réparti.

Ces considérations permettent de mieux préciser dans quelles conditions il faut aborder l'étude des affouillements.

a) Du fait de la lenteur du processus d'engravement d'une retenue, il est préférable, en général, de supposer qu'aucun matériau solide n'est évacué à l'aval;

b) La courbe naturelle hauteur-débit doit être corrigée en tenant compte du profil minimum d'érosion du lit aval;

c) Pour éviter une usure rapide du déversoir, à la suite d'un passage même occasionnel de gravier, il convient d'éliminer de son profil en long toute zone d'écoulement en dépression, dans lesquelles ces graviers pourraient ètre emprisonnés et faire subir des chocs répétés au béton avant de s'en échapper.

\section{B) CONSIDERATIONS THÉORIQUES}

Dans l'état actuel, les considérations théoriques ne permettent pas à elles seules de déterminer un ouvrage de dissipation d'énergie. Elles n'en restent pas moins indispensables pour poser les conditions auxquelles l'ouvrage doit satisfaire et définir les caractéristiques de la solution la plus simple : radier de réception horizontal, solution qui servira de base aux variantes envisagées au cours de l'étude sur modèle en évitant ainsi des tâtonnements inutiles.

\section{I. - Position du problème.}

On veut assurer le maintien d'un ressaut ordinaire au pied du barrage jusqu'à un certain débit $Q_{\mathrm{L}}$ de fréquence faible, par exemple le débit limite que peut évacuer le barrage sans exhaussement par rapport à la cote normale de retenue. Imaginons que l'on réalise simplement un radier horizontal au niveau du lit naturel.
On connait la courbe $h(Q)$ du niveau naturel aval, corrigée éventuellement, ainsi qu'il a été précisé ci-dessus, pour tenir compte de l'abaissement général du lit aval. On peut obtenir, par le calcul, la courbe $h^{\prime}(\mathrm{Q})$ donnant le niveau aval du ressaut ordinaire que l'on voudrait voir s'établir sur le radier. Les courbes $h$ et $h^{\prime}$ peuvent avoir les quatre positions relatives portées sur la figure 1 .

Si $h^{\prime} \leqslant h$, il se formera, selon la différence entre ces deux valeurs, un ressaut «ordinaire» (avec rouleau supérieur), « ondulé » (rouleaux supérieurs ou inférieurs), ou « noyé ». Dans ce dernier cas, il y aurait réduction du coefficient de débit du déversoir, ce qui devrait logiquement conduire à diminuer la largeur du barrage. Avec les deux autres types de ressaut, le risque d'affouillement est relativement faible: le radier horizontal au niveau du lit peut être considéré comme une solution d'avant-projet satisfaisante 
et dont on pourra partir pour la mise au point définitive.

Si $h^{\prime}>h$, le ressaut ne peut s'établir sur le radier. La lame d'eau quitte l'ouvrage en écoulement torrentiel et des afrouillements sont à craindre dont l'importance sera d'autant plus grande que le débit à partir duquel existe cette inégalité est plus élevé.

Ce sont donc les cas 3 et 4 de la figure 1 , ce dernier étant d'ailleurs le plus fréquent, qui nécessitent une étude approfondie.
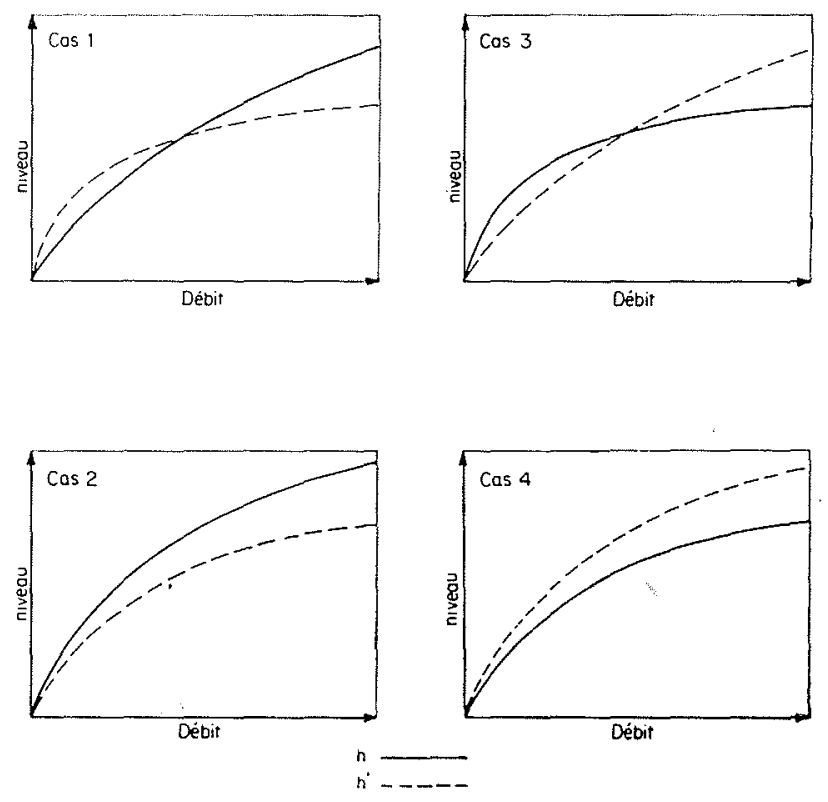

Fig. 1. - Comparaison du niveau naturel $h$ avec le niveau $h^{\prime}$ à laval d'un ressaut sur radier horizontal.

Pour que le ressaut s'établisse alors sur un radier horizontal de réception, on dispose de deux moyens :

- soit réduire le débit unitaire en augmentant la largeur du barrage,

- soit abaisser le niveau du radier par rapport au terrain naturel.

L'emplacement du barrage et le débit maximum étant fixés, le coût d'un ouvrage de retenue est, pour une largeur $L$ et un affouillement aval donnés, la somme des coûts du barrage, de l'endiguement amont et du bassin de tranquillisation nécessaire. Lorsque $\mathrm{L}$ augmente, le premier croît et les deux autres décroissent. Il existe donc une largeur optimum qu'il s'agit de déterminer.

Pour faire cette étude économique destinée à fixer les grandes lignes du projet, il faut savoir définir le bassin de tranquillisation connaissant les débits unitaires à évacuer. Le calcul que nous développerons ci-dessous suffit pour dégrossir une telle définition. Si, finalement, la poursuite de l'étude conduit à modifier le bassin de tran- quillisation calculé, la conclusion du calcul économique du barrage n'en sera pas sensiblement modifiée.

\section{II. - Calcul d'un radier horizontal à l'aval d'un barrage, de manière à maintenir le ressaut hydraulique au pied de l'ouvrage.}

a) Altitude DU Radier :

Le problème posé est le suivant:

Quel abaissement $x$ par rapport au seuil amont du barrage doit-on faire subir à un radier horizontal de réception réalisé au pied de l'ouvrage afin qu'un ressaut ordinaire se maintienne sur ce radier jusqu'au débit $Q_{r}$, choisi?

Les symboles utilisés sont définis sur la figure 2.

Les données sont les suivantes:

Q : Débit.

$L_{1}$ : Largeur d'écoulement supposée constante entre (0) et (1) (section rectangulaire).

$\mathrm{L}_{2}$ : Largeur d'écoulement dans la section (2).

$\lambda: \mathrm{L}_{1} / \mathrm{L}_{2}$.

$a_{\text {I }}$ : Hauteur d'eau aval rapportée à la cote du seuil amont du barrage (positif vers le haut).

On a, entre les sections (0) et (1):

$$
\mathrm{H}=h_{1}+\frac{V^{2}}{2 g}+\alpha \cdot \frac{V^{2}}{2 g}
$$

๙. $V^{2} / 2 g$ étant la perte de charge entre ces deux sections.

Ou si l'on pose $1+\alpha=1 / m$ (avec $m<1$ ):

$$
\mathrm{H}=h_{1}+\frac{1}{m} \frac{\mathrm{V}^{2}}{2 g}
$$

soit, d'après les conditions imposées :

$$
x+\frac{3}{2} h_{c}=h_{1}+\frac{1}{m} \frac{V^{2}}{2 g}
$$

D'autre part, si l'on applique le théorème d'Euler entre les sections (1) et (2), on obtient facilement l'équation suivante dite « des profondeurs conjuguées » :

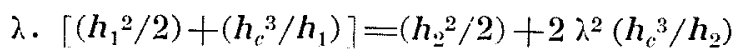

Pour que le ressaut se maintienne, il faut que le niveau à l'aval du ressaut (c'est-à-dire à l'extrémité du radier) soit au plus égal au niveau calculé à partir de la cote d'eau naturelle à l'aval de la fosse. Nous considérons ce dernier 


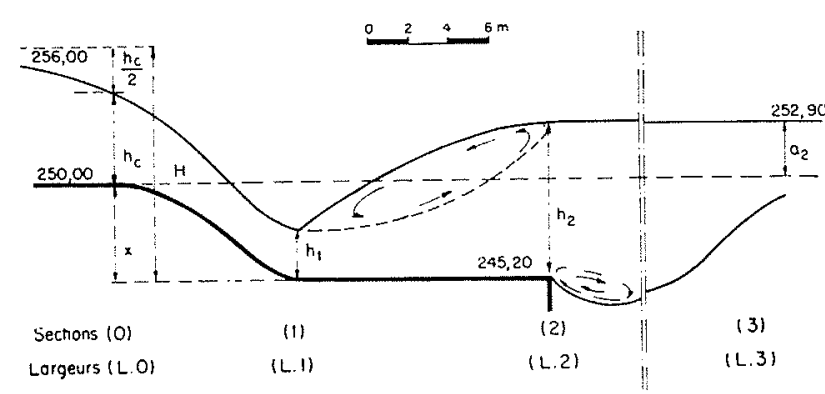

Fig. 2. - Calcul de l'altitude d'un radier hori rontal a l'aval d'un barrage pour maintenir le ressaut hydraulique au pied de l'ourrage.

Nota. - Les dimensions correspondent à l'écoulement du débit de $3.700 \mathrm{~m}^{3} / \mathrm{s}$ à travers le barrage de Cadarache.

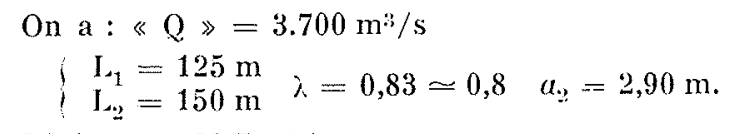

D'où : $q=29,7 \mathrm{~m}^{\ddot{3}} / \mathrm{s}$

$$
\begin{aligned}
& h_{c}=\sqrt[3]{q^{2} / g}=4,47 \\
& t_{2}=a_{2} / h_{c}=0,648
\end{aligned}
$$

Le diagramme donne : $u_{2} \leq-1,51$

$$
h_{.2}=u_{2} \times h_{c}=6,7 \tilde{5}
$$

Donc cote du radier : $252,90-6,75=\mathbf{2 4 6 , 1 5}$ (par défaut).

calcul comme superflu, car la perte de charge entre les deux sections est impossible à évaluer (nous reviendrons d'ailleurs sur cette question) et, de toute façon, faible en régime tranquille sur une courte distance.

On peut donc admettre, comme condition de stabilité dụ ressaut, l'équation suivante :

$$
h_{2}=x+a_{2}
$$

L'équation (1) peut alors s'écrire :

$$
h_{2}-a_{2}+\frac{3}{2} h_{c}=h_{1}+\frac{1}{m} \frac{\mathrm{V}^{2}}{2 g}
$$

Les équations (2) et (4) résolvent le problème. Elles se simplifient en rapportant les tirants d'eau à la profondeur critique $h_{\bullet}$. Soient :

$$
u_{1}=\frac{h_{1}}{h_{c}} ; u_{2}=\frac{h_{2}}{h_{c}} ; t_{2}=-\frac{a_{2}}{h_{c}}
$$

Il vient:

$$
\begin{gathered}
u_{2}-t_{2}+\frac{3}{2}=u_{1}+\frac{1}{2 m u_{1}^{2}} \\
u_{1}{ }^{2}+\frac{2}{u_{1}}=\frac{u_{2}^{2}}{\lambda}+\frac{2 \lambda}{u_{2}}
\end{gathered}
$$

Sur le diagramme en coordonnées $u_{1}, u_{2}$ de la figure 2, la courbe I représente l'équation (4') pour $t_{2}=0$ en prenant $m=0,9$ (valeur la plus

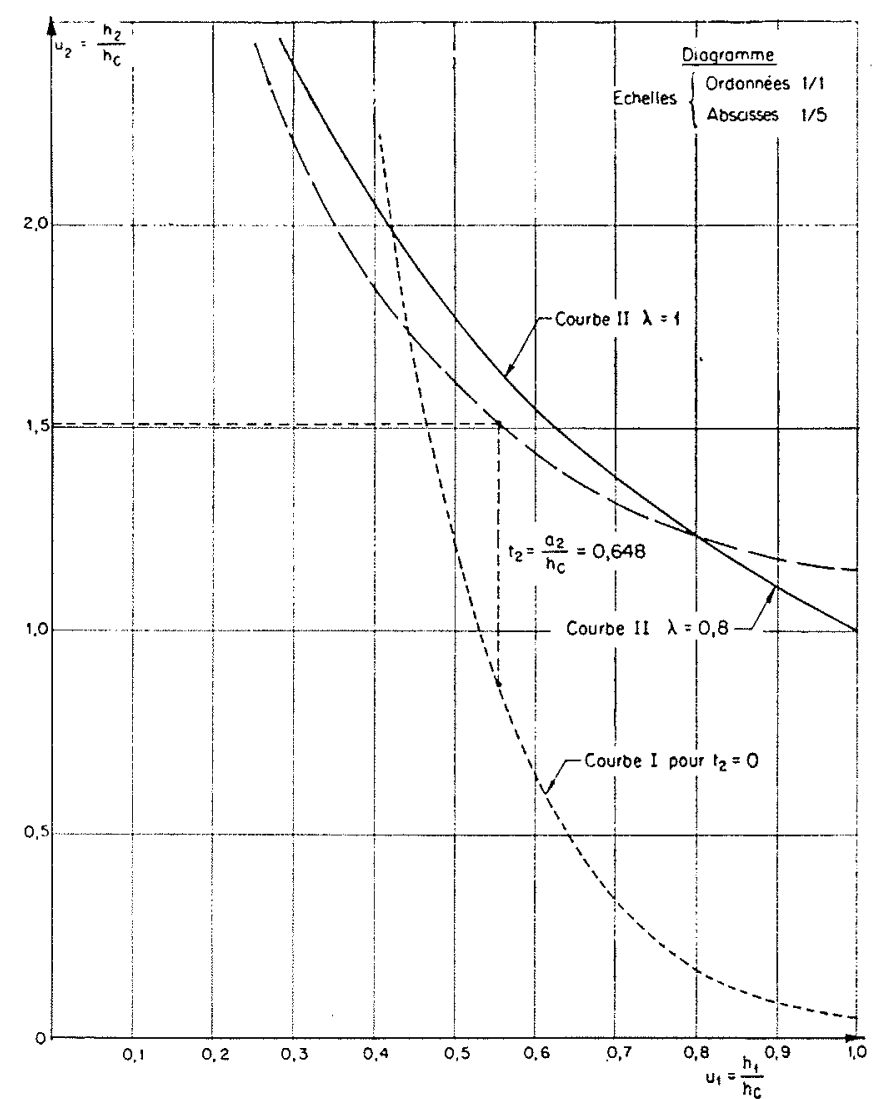

couramment admise) et les courbes II $\lambda$ l'équation $\left(2^{\prime}\right)$; pour $\lambda=1$, c'est l'équation classique :

$$
u_{1} u_{2}\left(u_{1}+u_{2}\right)=2
$$

Ce diagramme permet de déterminer la cote maximum cherchée du radier : pour une valeur connue de $a_{2}$ dans des conditions hydrauliques données permettant de calculer $h_{c}$, c'est-à-dire pour une valeur de $t_{2}=a_{2} / h_{6}$, on cherche le point de la courbe I distant de $t_{2}$ de la courbe II $\lambda$ des profondeurs réduites conjuguées. L'ordonnée $u_{2}$ de ce point permet de calculer $h_{2}=u_{2} h_{c}$; donc, la cote maximum du radier pour que le ressaut se maintienne.

On a porté sur la figure 2, à tilre d'exemple, l'application numérique au barrage de Cadarache (pour la crue centenaire $Q_{\mathrm{L}}=3700 \mathrm{~m}^{3} / \mathrm{s}$ ).

Le résultat obtenu a pu être vérifié expérimentalement. Les essais, dont la figure 3 donne quelques résultats, ont consisté à faire varier la cote du radier horizontal au pied du barrage et à évaluer pour chaque cote un ordre de grandeur du débit limite au-delà duquel le ressaut est chassé.

\section{b) Longuevr DU RADIER :}

La cote du radier étant ainsi définie, il reste 


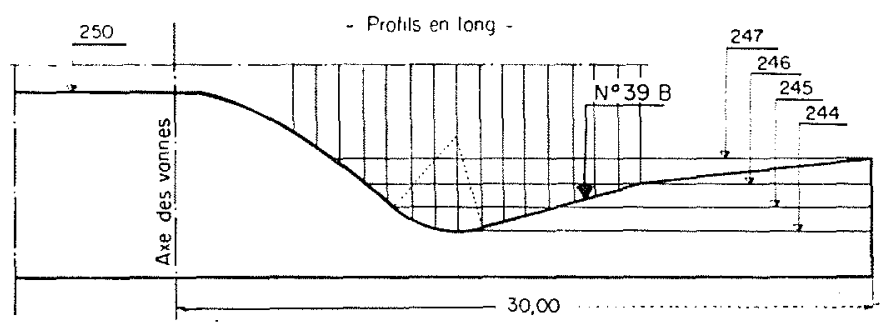

Tableau XII

Comparaison entre les radiers horizontaux $(Q=50 \mathrm{~L} / \mathrm{s} ; T=3$ heures $)$

\begin{tabular}{|c|c|c|c|c|c|c|c|}
\hline \multirow{2}{*}{$\begin{array}{c}\text { cote } \\
\text { du } \\
\text { radier }\end{array}$} & \multicolumn{2}{|c|}{ cote atheinte } & \multirow{2}{*}{$\begin{array}{l}\text { Volume } \\
\text { wen } \mid\end{array}$} & \multicolumn{4}{|c|}{ Ressaut } \\
\hline & axe & rives & & $\begin{array}{c}\text { Timite } \\
\text { amont (t) }\end{array}$ & $\begin{array}{c}\text { limite } \\
\text { aval }(1)\end{array}$ & $\begin{array}{l}\text { longueur } \\
\text { en m }\end{array}$ & $\begin{array}{c}Q, \text { limite de stabilité } \\
\text { en } \mathrm{m}^{3} / \mathrm{s}\end{array}$ \\
\hline 244 & 239 & 239 & 118,9 & 5 & 50 & 45 & $Q>8000$ \\
\hline 245 & 239 & 239 & 108,8 & $?$ & 36 & 29 & $7000<Q<8000$ \\
\hline 246 & 240 & 240.10 & 94,1 & 9 & 28 & 19 & $4500<Q<5000$ \\
\hline 247 & 239.25 & 240.35 & 89.6 & 4 & instable & $\longrightarrow$ & $Q<2500$ \\
\hline NO 39B & 242,00 & 241,75 & 70.1 & & & & $4000<Q<4500$ \\
\hline
\end{tabular}

Comparaison du radier horizontal à 246 et du radier $39 \mathrm{~B}$

\begin{tabular}{|c|c|c|c|c|c|c|}
\hline & $\begin{array}{c}\text { Durees } \\
\text { en }\end{array}$ & mulées & 3 & 12 & 18 & 24 \\
\hline \multirow{3}{*}{$\begin{array}{l}\text { Radier } \\
\text { horizontal } \\
\text { a } 246\end{array}$} & \multirow{2}{*}{$\begin{array}{c}\text { cote } \\
\text { ateinte }\end{array}$} & dansl'axe & 240. & 237.50 & 236.50 & 236.40 \\
\hline & & en riyes & 240.10 & 23825 & 238 & 236,80 \\
\hline & \multicolumn{2}{|c|}{ Volume Wen I } & 94.10 & 120.90 & 141.45 & 146.80 \\
\hline \multirow{3}{*}{ Radier $39 \mathrm{~B}$} & \multirow{2}{*}{$\begin{array}{l}\text { cote } \\
\text { atteinte }\end{array}$} & dans l'axe & 242 & 240 & 240 & 240 \\
\hline & & en rives & $24 t, 75$ & 2.39885 & 239,10 & 238,85 \\
\hline & \multicolumn{2}{|c|}{ Volume Wen l } & 70.1 & 1038 & 108,4 & 110,8 \\
\hline
\end{tabular}

Fia. 3. - Etude expérimentale des radiers horizontaux. Longueur du radier : $30 \mathrm{~m}$. Pas de rugosité. Piles triangulaires. à en fixer la longueur. Thériquement, cette longueur devrait être égale à celle du ressaut définie comme la distance entre les sections amont et aval où l'écoulement est et redevient uniforme, c'est-à-dire entre le début et la fin de la courbure des filets liquides.

Or, la longueur du ressaut a échappé jusqu'à maintenant à toute analyse mathématique précise.

On dispose seulement de formules empiriques qui ne définissent d'ailleurs le plus souvent et d'une manière assez contradictoire que la longueur du rouleau de surface, seule accessible aux mesures (1). C'est ainsi que, sur dix formules appliquées, les résultats varient entre 5 et $25 \mathrm{~m}$ pour une longueur mesurée de $20 \mathrm{~m}$. De plus, cinq d'entre elles donnent une longueur constante lorsque l'on abaisse le radier horizontalement tout en maintenant identiques les autres conditions expérimentales, ce qui est en contradiction avec les résultats de nos essais (cf. fig. 3). La formule la plus satisfaisante (et d'ailleurs la plus simple) nous semble être celle proposée par Smetana et d'après laquelle la longueur du ressaut serait égale à six fois sa hauteur.

Cette carence de la théorie du ressaut, en ce qui concerne ses caractéristiques longitudinales, est la raison pour laquelle on ne peut faire un calcul analogue au précédent sur un radier non horizontal. En effet, lorsque l'on applique l'équation des quantités de mouvement, il faut alors tenir compte de la composante de la pesanteur, donc connaitre la forme et la longueur du ressaut.

\section{C) ÉTUDE SUR MODÈLE}

Nous n'entrerons pas dans le détail des conditions que l'on doit respecter en choisissant l'échelle du modèle pour qu'il existe effectivement une similitude. Disons seulement qu'il faut d'abord satisfaire à la condition de similitude des écoulements à surface libre, à savoir que le « nombre de Reynolds » du modèle soit supérieur à la valeur limite d'écoulement à turbulence pleinement développée. Le phénomène étudié étant justement très turbulent, cette condition est facile à respecter.

Pour qu'il existe aussi une similitude des affouillements, c'est-à-dire du phénomène d'entraînement des matériaux, on peut choisir un matériau de même densité, de granulométrie et, si possible, de forme réduites selon l'échelle géométrique, à condition qu'il reste encore suffisamment grossier (rivières de montagne). On peut alors escompter de l'étude des résultats quantitatifs.

Si le matériau nécessaire s'avérait trop fin, il faudrait procéder différemment : réduire la densité du matériau, ou extrapoler à partir des profondeurs atteintes avec des matériaux de mème densité et de plus en plus fins jusqu’à la limite d'utilisation (2). Dans ce cas, la similitude absolue des affouillements devient plus discutable, malgré quelques recoupements satisfaisants déjà enregistrés. Cependant, si l'on choisit un mode opératoire identiquement reproductible, les ré-

(1) On peut en effet déterminer assez aisément cette longueur en localisant la section d'inversion des courants de surface.

(2) Scimemi [4] est l'auteur de cette méthode. Il l'a appliquée avec succès même dans le cas d'une fosse creusée dans le rocher : barrage de Conowingo. 
sultats obtenus restent valables en valeur relative et conduisent ainsi à un classement exact des différentes dispositions envisagées.

L'échelle étant fixée, il reste à choisir le mode de représentation du barrage et à déterminer la façon d'exploiter le modele.

\section{I. - Mode de représentation du barrage.}

Dans le cas d'un barrage à plusieurs passes, on se contente souvent de ne représenter en canal qu'une passe du barrage et une partie des passes adjacentes. Cette solution est évidemment moins onéreuse et permet d'étudier rapidement un nombre élevé de dispositions.

Il convient alors de prendre certaines précautions évidentes (cf. fig. 4, une vue du dispositif
- Rendre les déplacements de la vanne centrale parfaitement solidaires de ceux des demivannes latérales.

Mais, mème dans ces conditions, on peut se demander si les résultats obtenus seront identiques à ceux que l'on obtiendrait à l'aval du modèle représentant l'ensemble du barrage à la même échelle.

Cette question a fait l'objet d'une étude au cours des essais sur le barrage de Cadarache, pour lequel les deux modèles ont été construits à l'échelle de $1 / 50^{\circ}$.

En voici les conclusions :

Nous avons d'abord pu constater qu'il est bien indispensable de réaliser en canal, non seule-

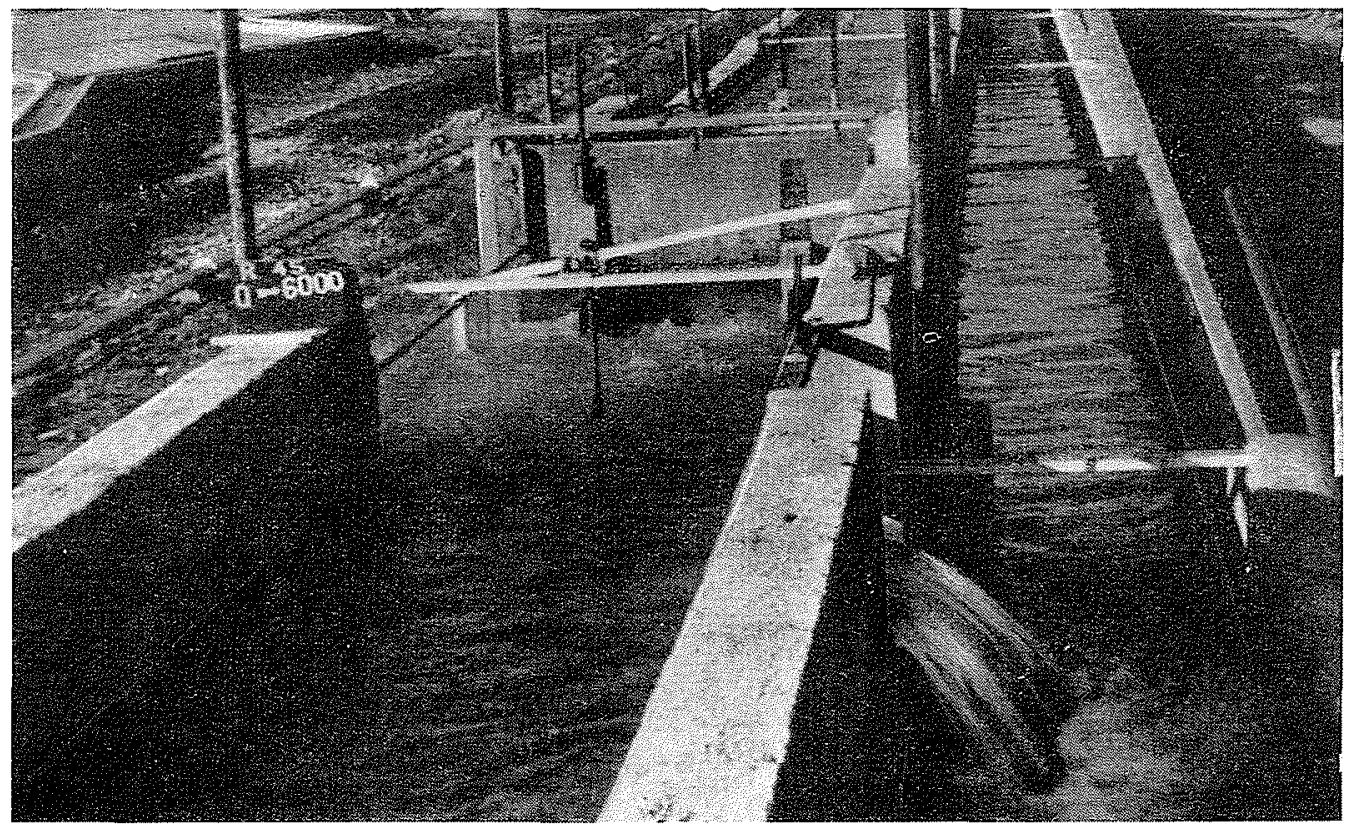

Fig. 4. - Vue générale du canal d'essai. Passage de la crue de $6.000 \mathrm{~m}^{3} / \mathrm{s}$ sur un radier horizontal à la cote 244 . Echelle des dẻbits : $1 / 52.600$.

expérimental pour l'étude du barrage de Cadarache) :

- Avoir à l'amont et surtout à l'aval du barrage une longueur de canal suffisante pour que le charriage s'effectue normalement; avant chaque essai, les fonds doivent être réglés à leur état initial avec le maximum de soin;

- Disposer à l'aval de la fosse d'affouillement une vanne latérale, permettant de corriger le niveau aval du canal de facon à le ramener au niveau naturel;

- Construire les deux piles avec une épaisseur telle que l'on respecte à peu près le coefficient d'étranglement du barrage; ment une passe complète, mais aussi une partie des deux passes adjacentes. Ainsi le relief obtenu à l'aval de la passe centrale n'est pas faussé par l'effet des parois du canal et il s'avère identique au relief relevé à l'aval d'une passe du barrage complet subissant les mêmes conditions d'écoulement.

Nous avons ensuite cherché à répondre à la question suivante :

La répartition du débit entre les vannes engendre-t-elle des effets particuliers au modèle d'ensemble?

A cet effet, on a suivi l'évolution de la fosse sur le modèle d'ensemble durant les essais d'engravement de la retenue de Cadarache (cf. fig. 5). 


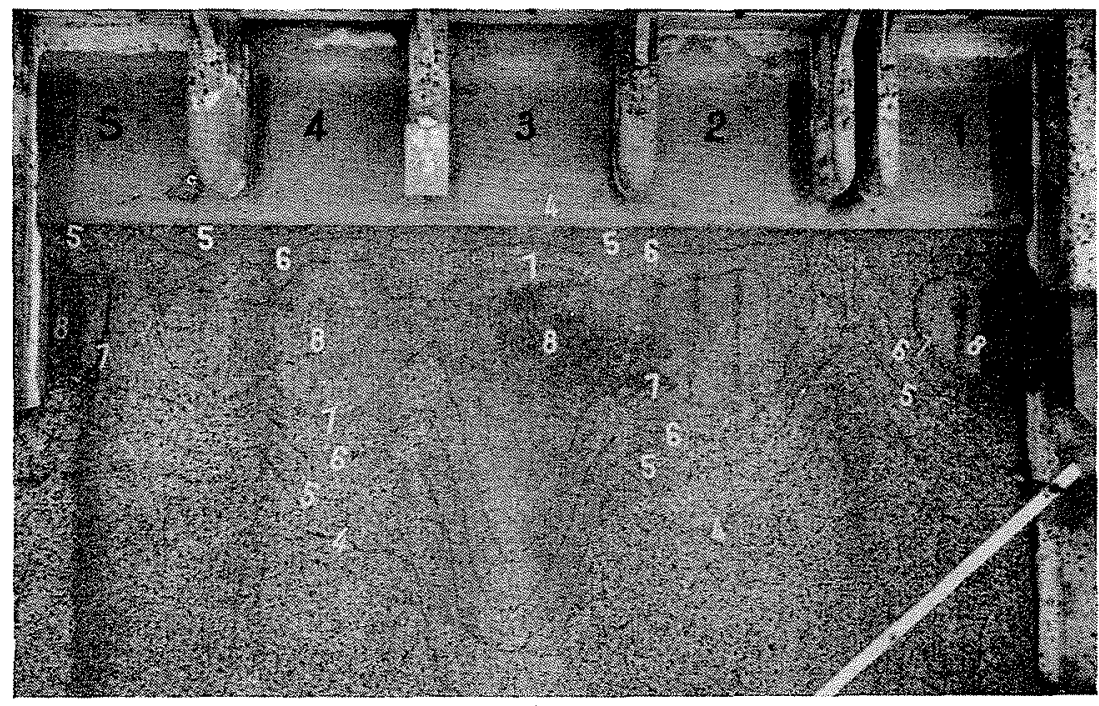

Après 16 ans

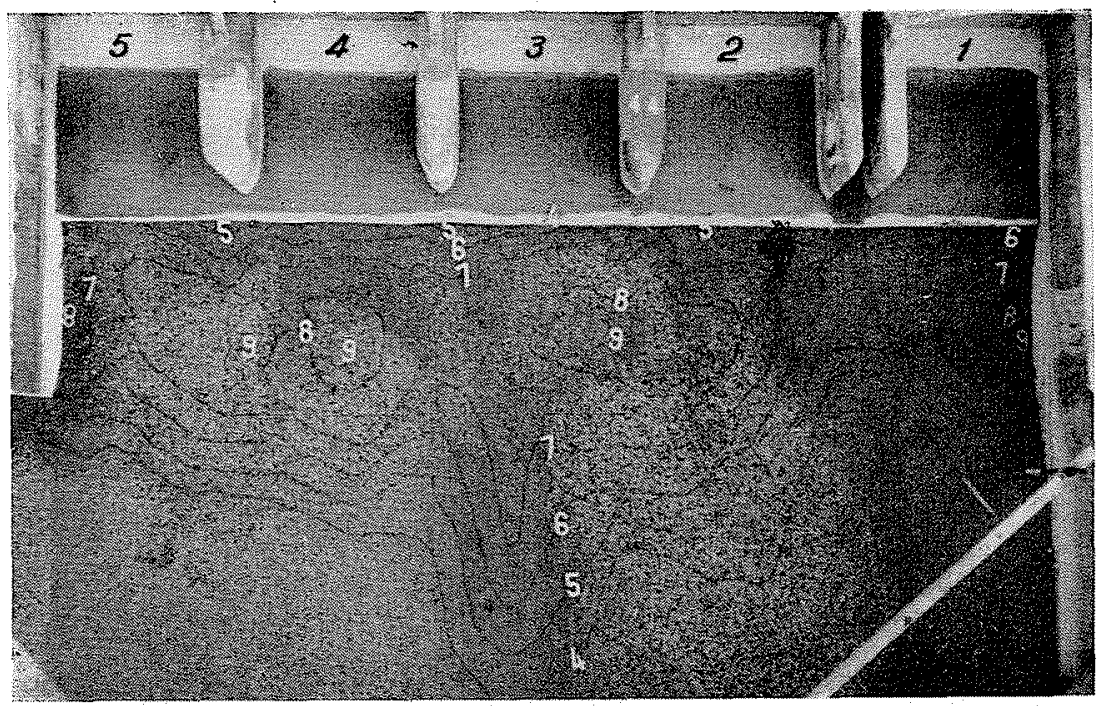

Après 18 ans

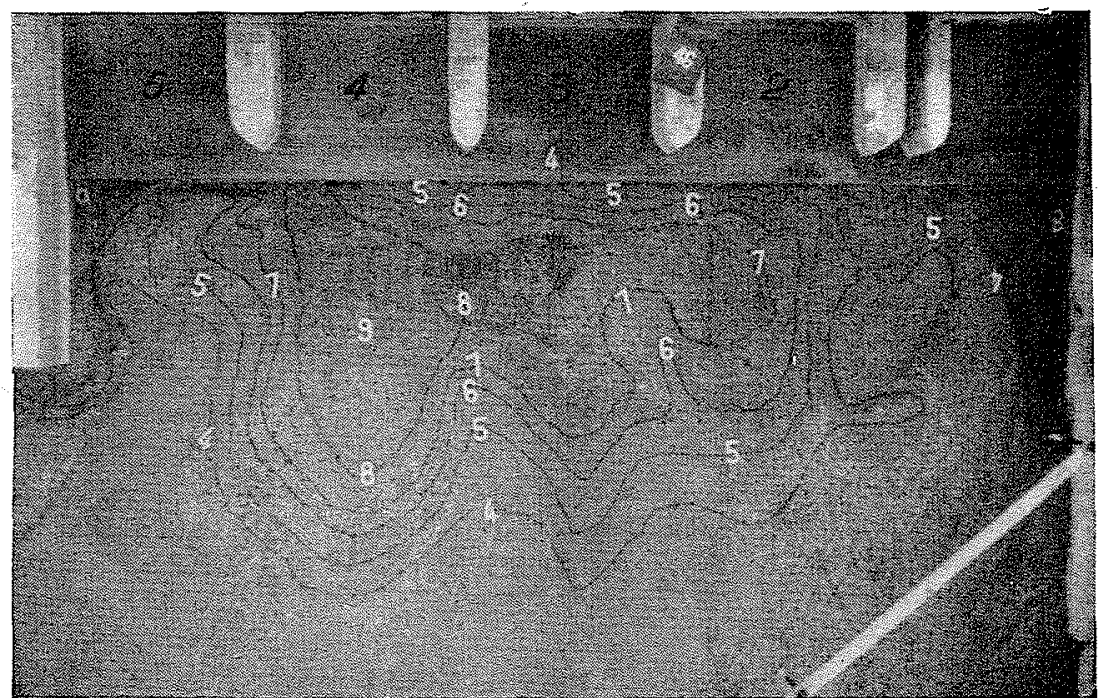

Après 22 ans
Fig. 5.

Evolution de la fosse sur le modèle d'ensemble pendant l'engravement de la retenue.

Lignes de niveau tous les mètres sur ces photographies et sur les suivantes (le zéro correspond it la cote 251 N.G.F.). 
Malgré l'application de consignes de manœuvres assez sévères (sur lesquelles nous reviendrons), on voit sur le dernier état après 22 ans que le pied des ouvrages, seuil du barrage et digues, subit localement des déchaussements qui atteignent les cotes extrêmes du centre de la fosse. Or, l'observation de ce phénomène particulièrement important échappait à l'étude en canal.

Il est curieux de constater, d'autre part, qu'il se produit surtout à l'aval des vannes les moins utilisées. Nous allons tenter d'en trouver une explication en analysant le processus d'évolution d'une fosse d'affouillement.

On peut y distinguer deux mouvements d'ensemble des matériaux de fond :

- A l'aval du point bas de la fosse (qui se déplace d'ailleurs très lentement vers l'aval), le sable subit une érosion d'intensité décroissante qui finit par s'annuler pratiquement lorsque l'ensemble de la contrepente est pavé en gros éléments;

- A l'amont du point bas, on observe sur le fond un mouvement général en sens inverse du courant liquide : le sable remonte par sauts successifs jusqu'au pied même du barrage, où il se trouve brusquement repris par bouffées et rejeté en suspension vers l'aval à son point de départ.

Ce second phénomène ne paraît pas beaucoup s'atténuer avec le temps. Les grains doivent s'user sous l'effet de la répétition de ce cycle fermé et les éléments fins mis en suspension, qui ont une vitesse de chute trop faible, finissent par s'échapper du rouleau et transiter vers l'aval, d'où une érosion de la partie amont de la fosse, mais qui doit être théoriquement très lente.

Il n'en est plus de même à l'aval d'une passe voisine d'une passe plus alimentée. Les dénivellations font naitre des courants de fond transversaux vers la passe voisine suivant un phénomène comparable à celui que l'on observe dans un coude de rivière. Les courants de fond perturbent le cycle normal des matériaux et provoquent donc l'érosion du seuil des passes peu utilisées, les matériaux ainsi arrachés se déposant dans la fosse des passes les plus utilisées, ou poursuivant leur transit vers l'aval. On pourrait $y$ remédier en prolongeant chaque pile par une cloison insubmersible jusqu'à l'aval de la fosse, mais cette disposition serait très onéreuse (1).

(1) A noter que cette disposition devient indispensable si, pour des raisons particulières, le radier d'une passe est à une cote différente de celle des autres passes (voir à ce sujet Butcher [9]).
Ceci explique aussi l'action nettement défavorable, mise en évidence par Schoklitsch [4], d'une dissymétrie ou d'un élargissement trop rapide des raccordements des culées du barrage aux berges de la rivière à l'aval.

Pour ce qui est de la validité d'une étude d'affouillement sur modèle partiel, on doit en retenir que le déchaussement du seuil ne sera pas fidèlement reproduit et qu'il peut atteindre localement la cote extrême de la fosse.

\section{II. - Mode opératoire sur modèle partiel.}

Ces conditions sur l'exécution du modèle partiel et l'interprétation des résultats étant admises, l'exploitation doit être conçue de manière à permettre la reproduction, aussi fidèle que possible et dans des conditions aussi proches que possible de la réalité, d'un essai-type servant de test pour chaque solution successivement envisagée.

a) Chotx des Caractéristiques de l'ESSAI-TYPE :

Une étude systématique de N'azir-Ahmad [5] a montré que la forme des profils d'égale profondeur, à l'aval d'un ouvrage déterminé, ne dépend pas du débit évacué, mais seulement de sa durée (2). Le plus rapide est donc de faire l'essai avec un débit élevé de fréquence faible pouvant aller jusqu'au débit maximum adopté pour le projet, la durée dépendant des phases de l'étude :

- Pour le dégrossissage du problème, quelques heures suffiront à discriminer les solutions entre elles;

- Pour la mise au point de la solution, l'essai devra être prolongé jusqu'à la stabilisation de la fosse afin d'obtenir la valeur extrême de la profondeur d'affouillement dont on se servira pour l'établissement du projet du parafouille et des ouvrages de raccordement aval (3)

\section{b) CHOIX DU CRITÈRE :}

A la fin de chaque essai-type, on photographie le relief de la fosse d'affouillement. On en déduit la cote maximum atteinte qui, d'après ce

(2) Voir aussi Scimeni [3] et Schoklitsch [4]. Plus précisément, d'après ces auteurs, les affouillements maximums susceptibles de se produire a l'aval des barrages ne dépendent ni de la nature du fond, ni du débit, mais seulement de la durée des débits et de la forme du barrage.

(3) Pour les essais sur le barrage de Cadarache, on a choisi un débit de $50 \mathrm{l} / \mathrm{s}$ pendant $24 \mathrm{~h}$, soit en nature $2.630 \mathrm{~m}^{3} / \mathrm{s}$ pendant une semaine. Or, le débit instantane de la Durance n'atteint ou ne dépasse ce chiff re qu'environ une fois tous les cing ans. 
qui précède, est un critère de comparaison valable pour le déchaussement à prévoir. A titre complémentaire, on peut aussi calculer le volume de la fosse qui caractérise l'énergie globale dépensée en érosion. D’après nos essais, ce second critère conduit au même classement que la cote maximum, mais donne une discrimination plus précise des solutions entre elles.

\section{D) DISPOSITIONS ADOPTÉES POUR LE BARRAGE DE CADARACHE}

\section{Principe APPLIQUÉ}

L'expérience montre qu'un radier horizontal de réception, disposé à une cote telle que la formation d'un ressaut ordinaire soit assurée, ne suffit pas en général à éviter les affouillements. Cela tient au fait qu'à l'extrémité de l'ouvrage et sur une assez longue distance à l'aval, d'une part, les vitesses sont variables en intensité et en direction; d'autre part, elles sont maxima au contact du fond et presque nulles en surface. C'est la régularisation des vitesses et le renversement de leur répartition verticale qui est $\dot{a}$ l'origine de la création de la fosse.

Parmi les formules proposées poưr l'évaluation des affouillements, l'une d'elles met bien ce phénomène en évidence. L'auteur [6] considère simplement que la profondeur atteinte est une fonction croissante de la différence d'énergie cinétique, entre les sections (2), située à l'extrémité du radier, et (3), correspondant à la première section de lit naturel après la fosse, c'est-à-dire de l'expression $q\left(\alpha_{2} V_{2}{ }^{2}-\alpha_{3} V_{3}{ }^{2}\right)$, où $\alpha_{2}$ et $\alpha_{3}$ sont les coefficients cinétiques. Or, $\alpha_{2}$ peut atteindre à l'aval d'un ressaut des valeurs très supérieures à celle qu'il présente en écoulement à turbulence normale. Ainsi, pour une même vitesse moyenne à l'extrémité du radier, on peut avoir des affouillements très différents selon $\alpha_{2}$, c'est-à-dire selon le degré de non-uniformité de l'écoulement à l'extrémité du radier.

La stabilisation d'un ressaut ordinaire au pied de l'ouvrage n'est donc pas une condition suffisante à la résolution du problème des affouillements. Il faut aussi rechercher des moyens propres à réaliser à l'extrémité du radier un écoulement de même nature que l'écoulement naturel dans la rivière.

En première analyse, on peut jouer sur le profil en long et la rugosité du radier.

Le principe directeur que nous avons appliqué pour déterminer ces facteurs au cours des essais a été le suivant : nous avons admis que les conditions optima devaient être atteintes avec les caractéristiques géométriques qui limitent au minimum la discontinuité imposée par le barrage aux conditions naturelles d'écoulement.
Cela nous a conduit à éliminer un certain nombre de solutions classiques du type profil en cuvette terminé par un seuil en saillie continu ou discontinu. Au passage d'un tel seuil, l'écoulement se trouve brusquement accéléré et les lignes de courant deviennent instables en direction et intensité. Nous avons d'ailleurs pu vérifier, par des essais comparatifs, que le rendement de tels radiers est très inférieur à celui de la solution finalement adoptée. Nous avons constaté aussi que l'on n'arrive pas à améliorer les résultats en faisant varier les dimensions (longueur et profondeur) de la cuvette qui précède le seuil aval.

Le mème principe de continuité trouvera aussi son application dans les études complémentaires décrites ensuite et relatives à la forme en plan des piles et à la nature des enrochements de protection.

Nous traiterons, pour terminer, du problème des consignes de manœuvre d'un barrage à plusieurs passes.

Dans toutes les solutions envisagées, on a conservé pour la partie amont de l'ouvrage, le même profil en long constitué par un seuil horizontal sous les vannes, puis un profil déversant du type Creager, et l'étude a porté sur l'ouvrage de réception proprement dit.

\section{I. - Etude du profil en long du radier (cf. fig. 6).}

Nous avons réalisé, dans cette étude, des radiers redressant la veine liquide à l'aide de profils constitués d'une seule, puis de deux contrepentes, en faisant varier systématiquement les caractéristiques géométriques de ces profils. Le radier le plus satisfaisant a été ensuite comparé au radier horizontal optimum résultant d'une série d'essais que nous avons déjà mentionnée.

Sans entrer dans une analyse détaillée des résultats, nous noterons seulement que la fosse la moins profonde de chaque série correspond au radier assurant le meilleur compromis entre 


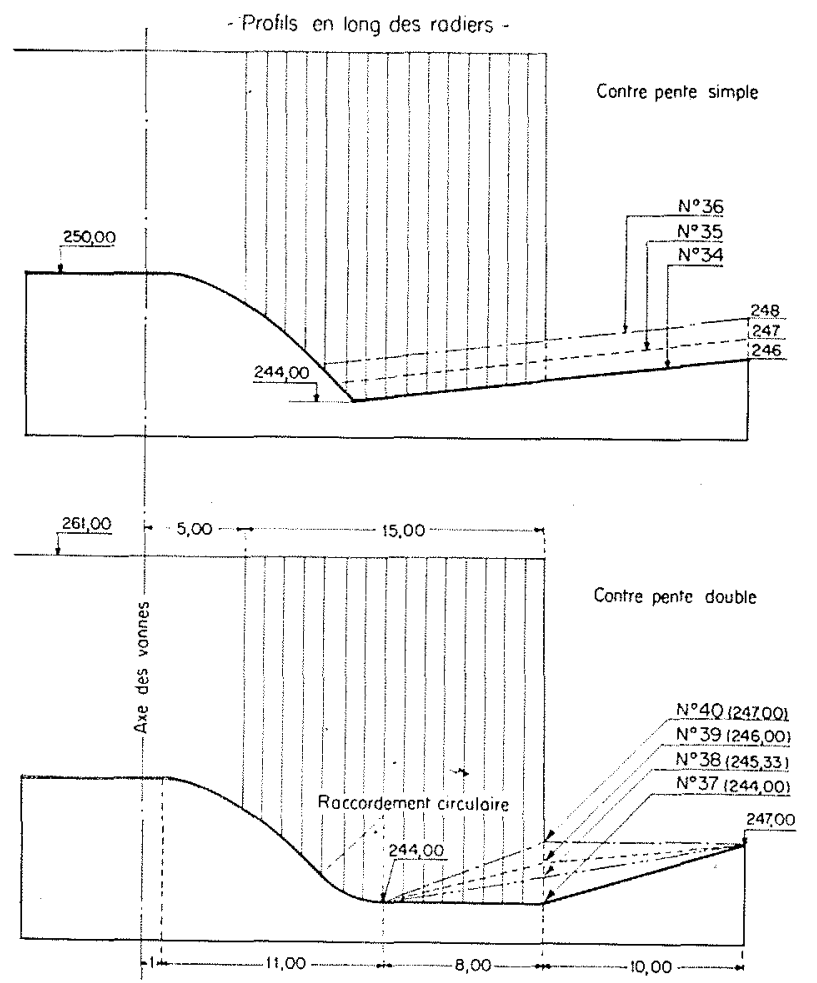

\begin{tabular}{|c|c|c|c|c|c|}
\hline \multirow{4}{*}{ Tableau: I } & \multirow{2}{*}{\multicolumn{2}{|c|}{\begin{tabular}{l|l} 
Radier N: Rugosité \\
\end{tabular}}} & Cote & tteinte & Volume \\
\hline & & & $\Delta \times e$ & Rive & $\checkmark$ en \\
\hline & $34-$ & 3.2 .1 & 242.00 & 242.50 & 11.4 \\
\hline & $34 \mathrm{~b}$ & 0 & 240,50 & 241.00 & 31.6 \\
\hline \multirow{4}{*}{ Contre pente simple } & $35 \cdots \cdots$ & 3.2 .1 & 242.50 & 242.00 & 13,6 \\
\hline & $36-\cdots$ & 3.2 .1 & 243.50 & 243.00 & 1.25 \\
\hline & $36 c$ & 3.3 .3 & 24350 & 24200 & 11,35 \\
\hline & $38 \ldots-\ldots$ & 3.2 .1 & 24300 & 242.00 & 9.00 \\
\hline
\end{tabular}

Fig. 6. Etude du profil en long du radier.

Longueur du radier : $30 \mathrm{~m}$. Rugosité additionnelle décroissante. $\mathrm{T}=3 \mathrm{~h} \quad Q=50 \mathrm{l} / \mathrm{s}$.

Piles triangulaires (cf. figure 11).

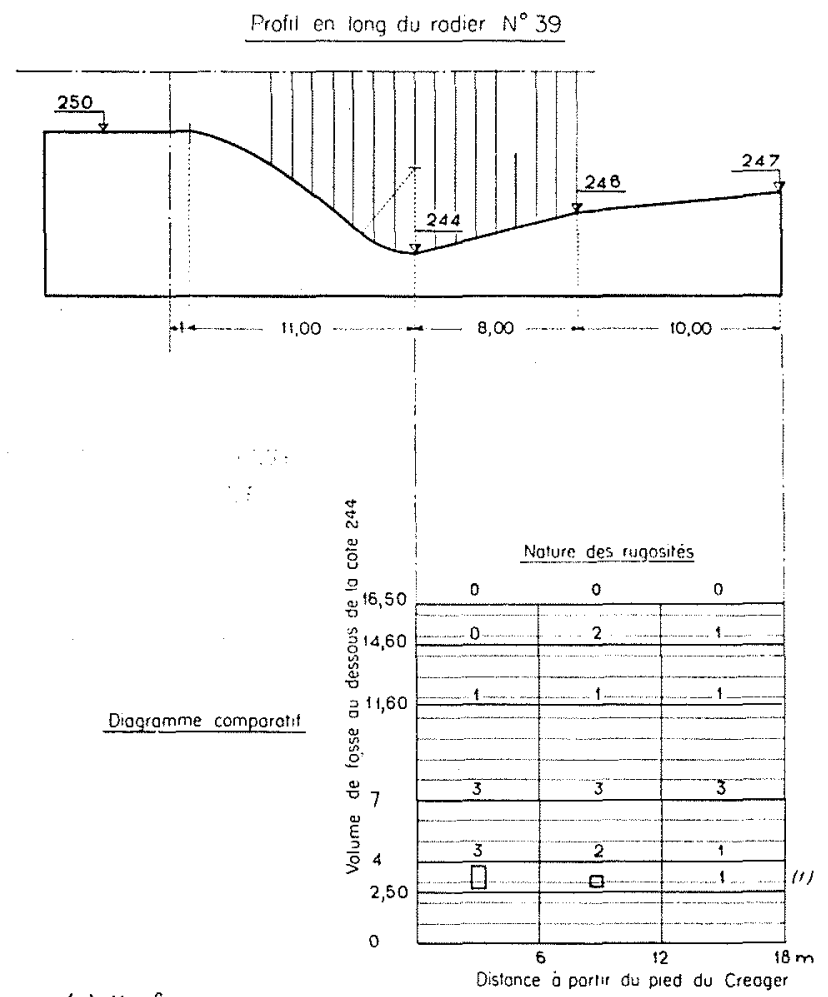

Légende

(1) Le ressaut est instable

(2) Le ressaut ne se maintient pas sur le seull

(1) Voir figre 8

Fıc. 7. - Variation de la rugosité additionnelle sur le radier $n^{\circ} 39$. $\mathrm{T}=3 \mathrm{~h} \quad \mathrm{Q}=501 / \mathrm{s}$ 
la stabilité du ressaul et la progressivité de l'écoulement entre l'amont et l'aval.

Nous avons été finalement conduits au profil $n^{*} 39$ (cf. fig. 7) avec lequel la veine, après son déversement sur le Creager jusqu'à une cote inférieure à la cote du radier horizontal optimum, est ensuite redressée par deux plans successifs d'inclinaison décroissante. Ce profil marque un progrès très sensible par rapport au radier horizontal (cf. fig. 3 : la fosse rapportée à la cote du radier horizontal est passée de $9,60 \mathrm{~m}$ à $6 \mathrm{~m}$ ). Il est aussi nettement plus satisfaisant que le type de radier à profil concave que l'on rencontre couramment dans les projets (profil n“37, le plus mauvais de la série).

Il est probable que l'on pourrait encore améliorer les résultats en augmentant le nombre de pentes successives. On serait ainsi conduit à la limite à choisir un profil comportant de l'amont ver's l'aval :

1" Une courbe de raccordement au profil Creager prolongée jusqu'à une cote suffisante pour maintenir le ressaut jusqu'à des débits élevés;

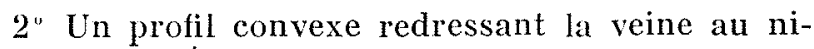
veau dú fond naturel'aval.

Nous avons constaté d'ailleurs que c'est la forme à laquelle on arrive si l'on réduit l'échelle des longueurs d'un profil naturel de fosse. Pour des raisons de simplicité de construction, on n'a pas cherché de progrès dans ce sens. C'est donc avec le profil $n^{\circ} 39$ que l'on a effectué les diverses études suivantes.

\section{II. - Etude systématique de la rugosité sur le radier $n^{\circ} 39$.}

L'étude précédente sur le profil en long a été effectuée en présence d'une rugosité choisie $a$ priori (rugosité 321 ). En voici la description : la longueur de radier à partir du pied du Creager a été divisée en trois parties égales sur lesquelles on a réalisé les rugosités suivantes, décroissantes de l'amont vers l'aval :

-- Rugosité 3: gravillon roulé, entre 700 et $500 \mathrm{~mm}$ de diamètre à l'échelle réelle;

-.- Rugosité 2 : gravillon roulé, entre 500 et $300 \mathrm{~mm}$;

- Rugosité 1: grain de sable anguleux, entre 300 et $100 \mathrm{~mm}$, c'est-à-dire de dimension comparable aux plus gros galets que l'on rencontre en Durance.

Il est donc apparu nécessaire, après avoir fait choix du profil en long du radier, de revenir plus systématiquement sur sa nature superficielle. On a commencé par rechercher sur un même profil (le profil n" 39) la rugosité optimum. On a ensuite obtenu confirmation du progrès réalisé par l'addition sur le radier lisse de cette rugosité optimum, d'une part en faisant varier la longueur du radier, d'autre part en procédant à des essais de longue durée avec une même longueur de radier.

a) VARIATION DE LA RUgOSITÉ SUR LE PROFIL $\mathrm{N}^{\circ} 39$ :

1' Un certain nombre d'essais ont été d'abord effectués à l'aide des trois rugosités en gravillon et sable que nous venons de définir (cf. fig. 7).

L'examen des reliefs obtenus appelle la remarque préliminaire essentielle suivante : si l'on porte sur un même graphique tous les profils en long de fosse, on constate que leur point bas reste toujours à la mème distance du seuil aval, à l'inverse des essais précédents au cours desquels on faisait varier le profil du radier. Cela tendrait à prouver que, pour des conditions hydrauliques données, la rugosité agit seulement sur l'intensité des phénomènes tourbillonnaires qui engendrent la fosse, alors que le profil en long du radier en détermine la répartition.

Le diagramme de la figure 7 donne le classement, d'après son degré d'efficacité, de chaque type de rugosité réalisé. On en tire les constatations suivantes :

- L'effet de la rugosité additionnelle est particulièrement sensible sur la pente amont (rugosités 321,021 et 000 );

- L'effet d'une rugosité additionnelle constante augmente avec la valeur de cette rugosité (rugosités $3 \quad 3 \quad 3,111$ et 000 );

- Une rugosité décroissante dans le sens de l'écoulement est préférable à la rugosité maximum réalisée sur l'ensemble du radier (rugosités 321 et $3 \quad 3 \quad 3$ ).

Finalement, c'est avec la rugosité additionnelle décroissante 321 , choisie a priori et répondant le mieux à ces observations, que l'on a obtenu les résultats les plus satisfaisants.

$2^{\circ}$ On a ensuite réalisé, sur le même radier 39 , une rugosité additionnelle décroissante, à l'aide de parallélépipédes présentant un plan diagonal parallèle à la direction de l'écoulement et répartis uniformément sur le radier, comme l'indique la figure 8 .

Le progrès enregistré par rapport à la rugosité optimum précédente 321 s'avère comme à peine sensible (cf. tableau III de la fig. 7). 

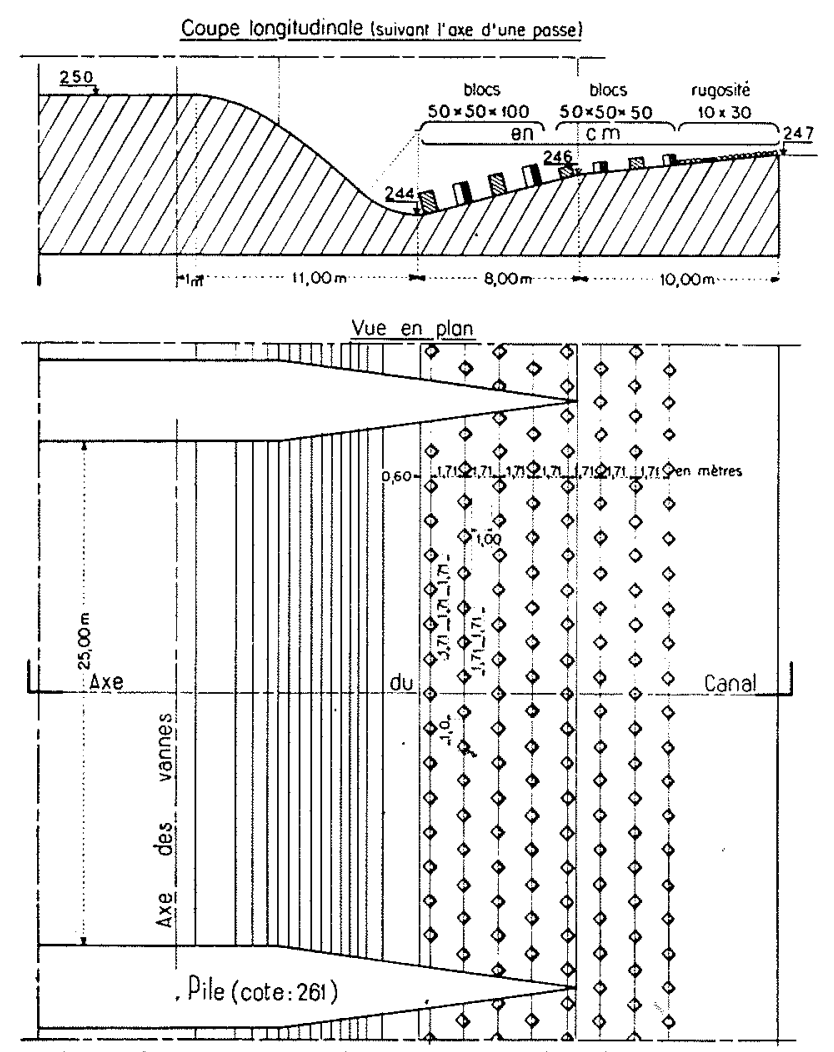

FIG. 8. - Radier à ragosité additionnelle décroissante en Jloes préfabriqués (rugosité adoptée pour le barrage de Cadarache).

\section{1 -Radiers sans rugosite}

\begin{tabular}{|c|c|c|c|c|}
\hline \multirow{3}{*}{ Tableau:R- } & \multirow{2}{*}{\begin{tabular}{|c}
$\begin{array}{c}\text { Radier } \\
\mathrm{N}:\end{array}$ \\
\end{tabular}} & \multicolumn{2}{|c|}{ Cote atteinte } & \multirow{2}{*}{$\begin{array}{l}\text { Volume } \\
v \text { en } l\end{array}$} \\
\hline & & Axe & rives & \\
\hline & 42 & 240,60 & 240,60 & 38,00 \\
\hline & 398 & 24200 & 241,75 & 16,50 \\
\hline & 41 & 241,65 & 24150 & 15,10 \\
\hline
\end{tabular}

\section{2-Radiers avec rugosité}

\begin{tabular}{|c|c|c|c|}
\hline $42 \mathrm{~A}$ & 243,20 & 241,90 & 15,40 \\
\hline $43 \mathrm{~A}$ & 243,25 & 242,70 & 3,90 \\
\hline $39 \mathrm{E}$ & 243,40 & 243,20 & 2,50 \\
\hline \hline $41 \mathrm{~A}$ & 243,50 & 243,35 & 2,10 \\
\hline
\end{tabular}

Rugosité : Ractiess: $424 ; 434 ; 38 \varepsilon$ : rugosué en blows selon la figure 5

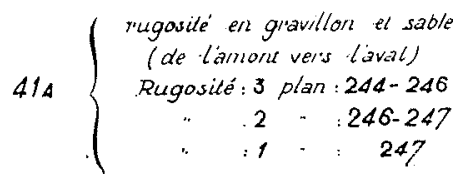

Il est cependant probable que l'on pourrait améliorer ce résultat en jouant sur les dimensions, la forme et la répartition des éléments rugueux: par exemple, en plus de la répartition longitudinale de la rugosité dont on a démontré l'utilité, sa répartition transversale devrait aussi permettre de limiter le déchaussement du seuil que l'on observe toujours à l'aval des piles.

Mais l'intérêt essentiel de cet essai était de montrer la grande latitude dont on dispose pour réaliser pratiquement sur un radier une rugosité qui renforce notablement l'efficacicé de son profil. On peut, par exemple, envisager de le revêtir en plaques préfabriquées, simplement juxtaposées, de manière à éviter la transmission des vibrations, et comportant chacune un ou plusieurs blocs de forme géométrique simple.

b) VARIATION DE LA LONGUEUR DU RADIER AVEC. OU SANS RUgOSITÉ :

L'ensemble de ces essais a été effectué à partir du radier $\mathrm{n}^{\circ} 39$, sans, puis avec rugosité. Les profils et rugosités réalisés sont portés sur la figure 9.

Cette figure montre que:

- L'allongement du radier dans des limites raisonnables, en présence ou en l'absence de rugosité, améliore peu les résultats.

- Par contre, pour une même longueur de radier, l'amélioration due à la rugosité est très sensible.
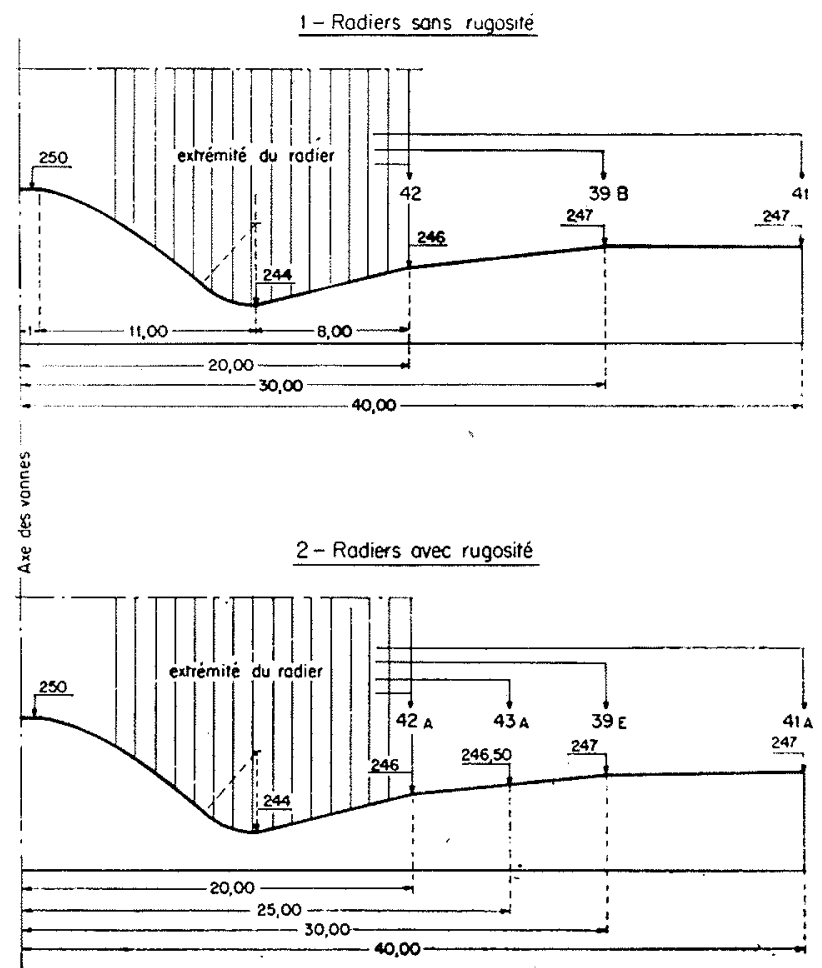

Fig. 9. - Variation de la longueur à partir du radier $n^{\circ} 39$ avec ou sans rugosité. 
Finalement, le radier avec rugosité le plus court $(20 \mathrm{~m})$, qui est évidemment le plus mauvais de sa série, reste cependant équivalent, tant au point de vue importance de la fosse que position de son point bas, au radier sans rugosité de longueur double, le meilleur de sa série. On peut donc affirmer que, pour limiter l'affouillement à une valeur donnée, la mise en place d'une rugosité apparaît comme plus économique que l'allongement nécessaire du radier (pour le barrage de Cadarache le rapport de prix serait de $1 / 4)$.
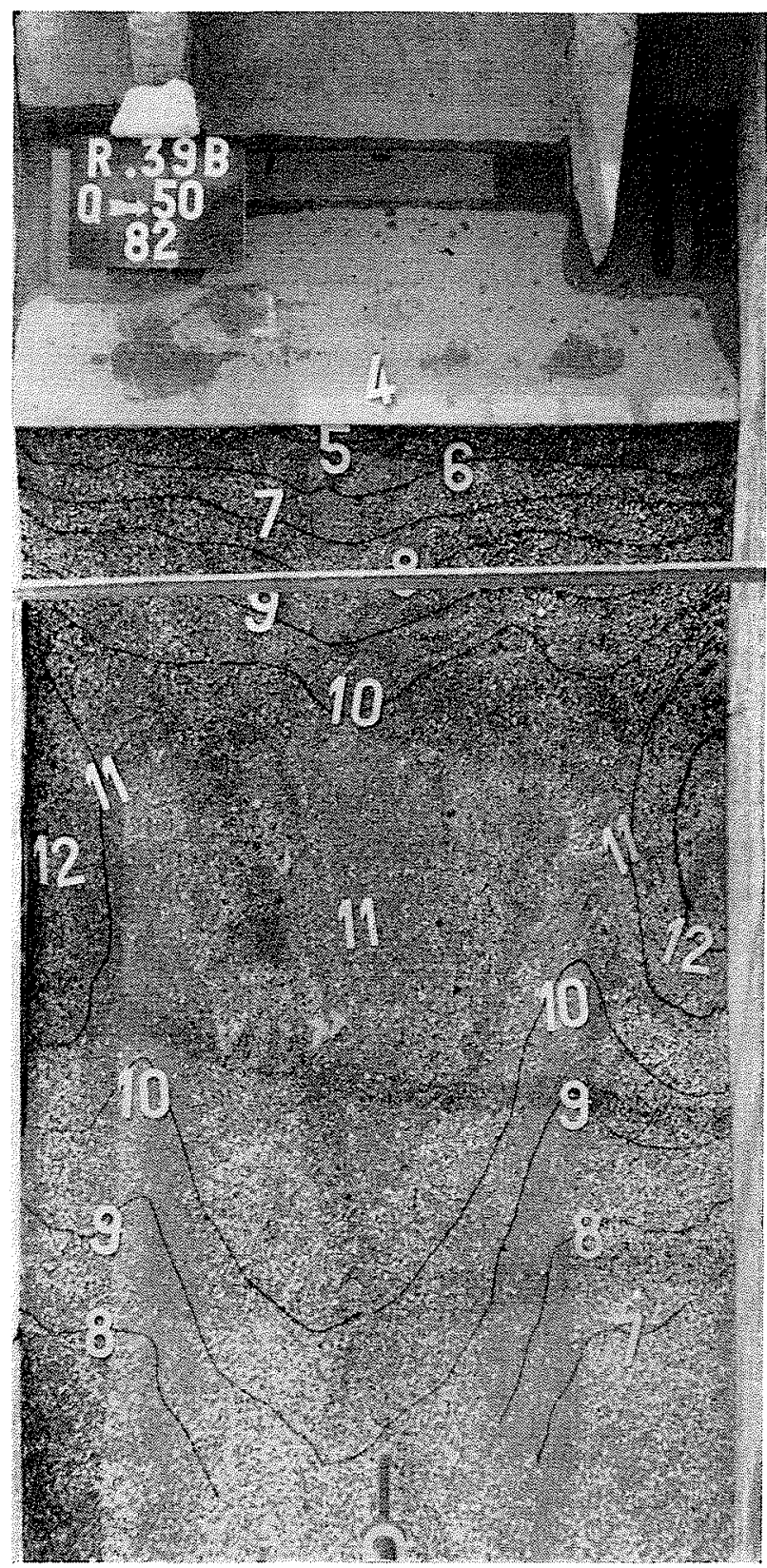

c) ESSAIS DE LONGUE DURÉE SUR LE RADIER N ${ }^{\circ} 39$ SANS RUgOSITÉ, PUIS AVEC RUGOSI'TÉ EN BLOCS :

L'essai-type utilisé jusqu'à maintenant était trop court ( 3 heures) pour que la fosse atteigne un état même voisin de son équilibre. Il convenait done de savoir dans quelle mesure le progrès enregistré, grâce à la rugosité, se conservait durant toute l'évolution de la fosse. A cet effet, on a procédé sur le profil $\mathrm{n}^{\circ} 39$ sans rugosité, puis avec rugosité en blocs, à un essai-type de
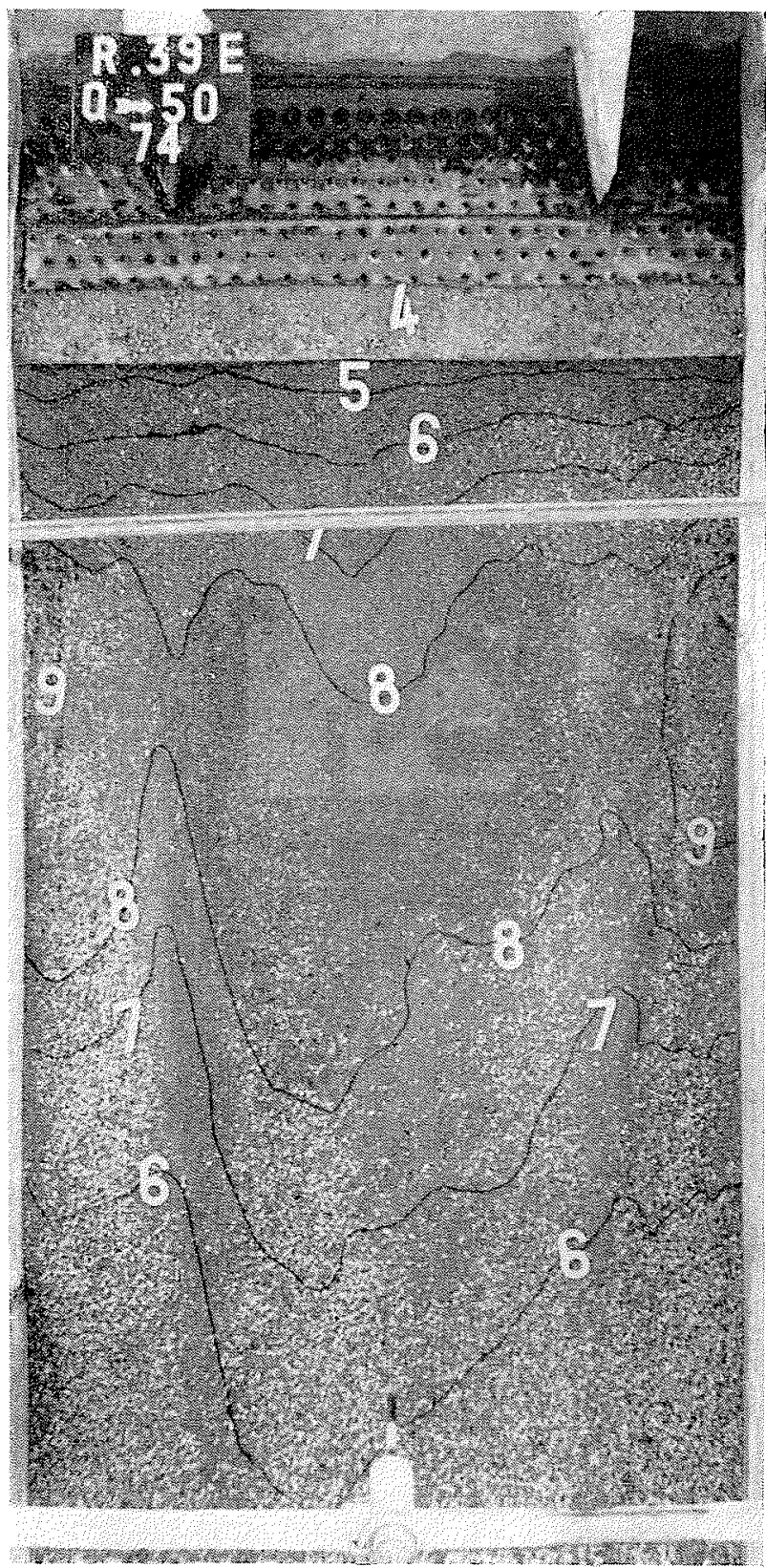

$b$

Frg. 10. - Effet de la rugosité avec le profil $n^{\circ} 39$. Piles triangulaires.

Essai-type de longue durée : $a$ ) sans rugosité; $b$ ) avec rugosité en blocs. 
durée suffisante pour que l'équilibre puisse être considéré comme atteint ( 33 heures au total). Les photos de la figure 10 donnent le relief de la fosse à la fin de chaque essai. Elles démontrent que l'amélioration due à l'adjonction d'une rugosité sur le radier est encore plus sensible à l'équilibre: la profondeur atfeinte all-dessous du seuil est en effet réduite de près de $50 \%$.

\section{d) Conclusion :}

Il est évident que la transposition quantitative de l'ensemble de ces résultats sur l'influence de la rugosité est discutable. Il reste cependant la conclusion qualitative suivante, certainement valable dans la réalité : un ouvrage de dissipation d'énergie doit présenter une forte rugosité, dont la valeur diminue de l'amont vers l'aval pour devenir, à son extrémité, de l'ordre de la rugosité du fond naturel.

Ces conclusions empiriques sont à rapprocher d'une formule donnant la longueur du ressaut [7], établie par un raisonnement évidemment très théorique, et d'après laquelle cette longueur varierait comme le carré du coefficient de rugosité. Sans que cette loi puisse être prise comme formellement exacte, elle confirme cependant l'importance de la rugosité d'un radier sur son efficacité.

\section{III. - Influence de la forme des piles.}

On a seulement envisagé les trois types de piles définis sur la légende de la figure 11, à savoir :

- Des piles « cylindriques », c'est-à-dire d'épaisseur constante avec extrémité demicirculaire;
- Des piles «triangulaires », plus courtes, qui avaient été choisies pour tous les essais antérieurs;

- Des piles « semi-triangulaires », de longueur et de variation d'épaisseur intermédiaire entre les deux types précédents.

Les figures 11 et 12 donnent les résultats de l'essai-type de longue durée appliqué avec le radier $39 \mathrm{~B}$ en présence de chacune de ces piles. Ces résultats démontrent sans équivoque l'importance de la forme en plan des piles sur les affouillements : les piles triangulaires donnent la fosse la moins profonde. La profondeur maximum atteinte augmente de $1,50 \mathrm{~m}$ avec les piles semi-triangulaires et de $4 \mathrm{~m}$ avec les piles cylindriques.

Il apparait ainsi que l'on a intérêt à réduire au minimum la longueur et l'épaisseur des piles à partir de la remontée du radier. On favorise en effet de cette manière la stabilisation du ressaut sur l'ouvrage. Cette considération devrait, dans certains cas, venir à l'actif de l'utilisation de la précontrainte pour les piles d'un barrage à plusieurs passes.

\section{IV. - Protection par tapis en enrochement.}

\section{a) Considérations THÉonigues :}

Malgré toute la confiance que l'on peut attribuer au projet de l'ouvrage de dissipation, il est couramment jugé préférable, à titre de sécurité supplémentaire, de disposer à partir du pied de louvrage un tapis de protection en enrochement. Or, si curieux que cela paraisse, il ne semble pas exister en France, pour l'application

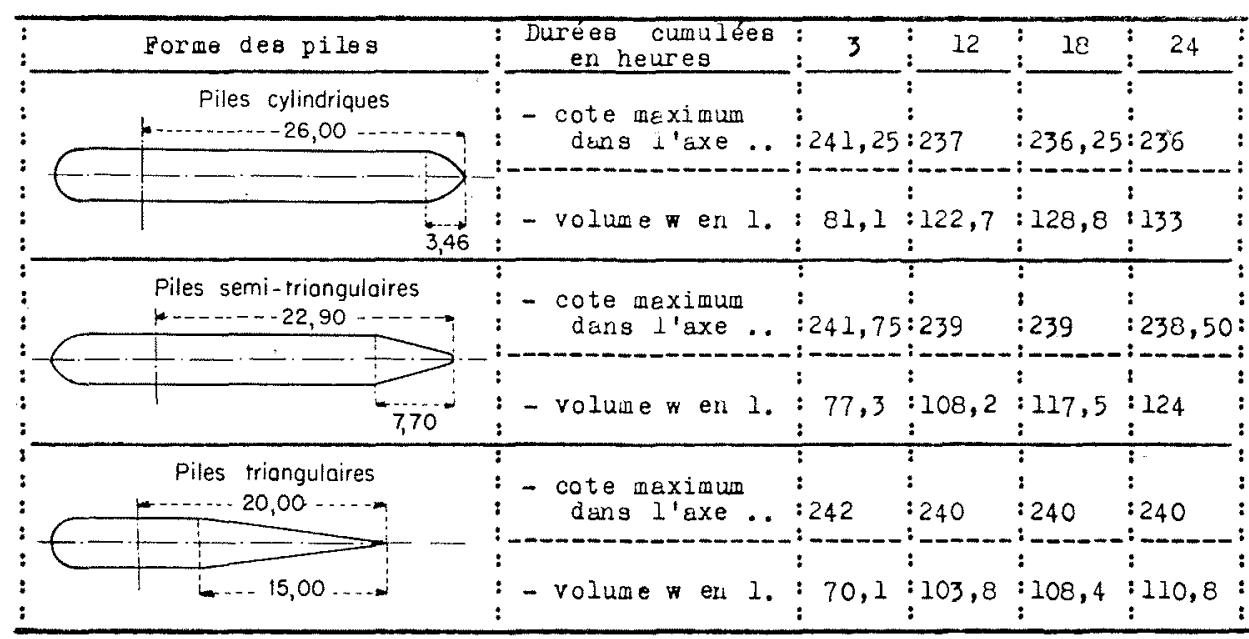

Frg. 11. - Influence de la forme en plan des piles, Profil $\mathbf{n}^{\circ} 39$ sans rugosité (39 B) Essai-type de longue durée. $Q=501 / \mathrm{s}$ 

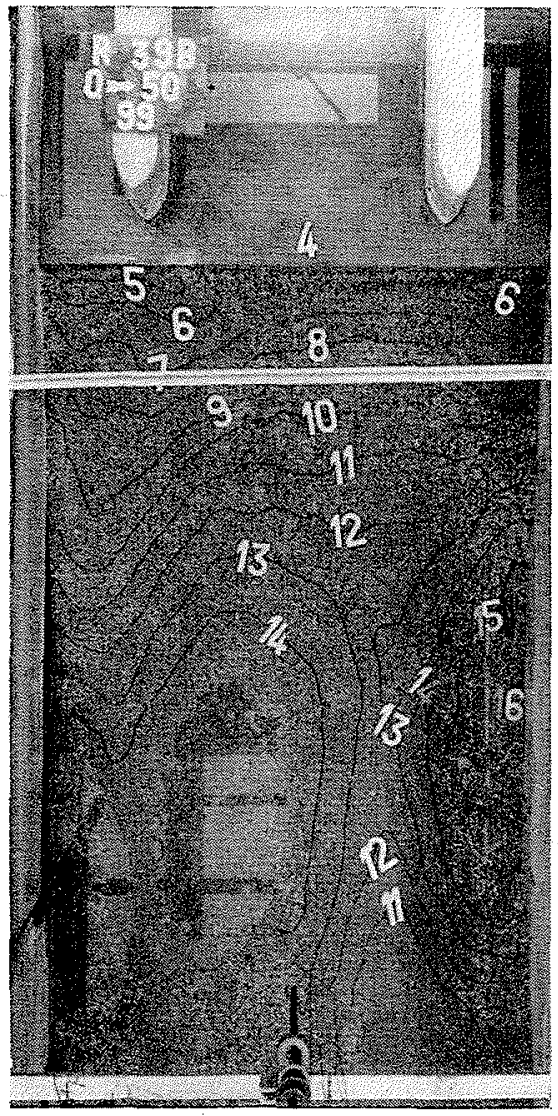

a) piles cylindriques.

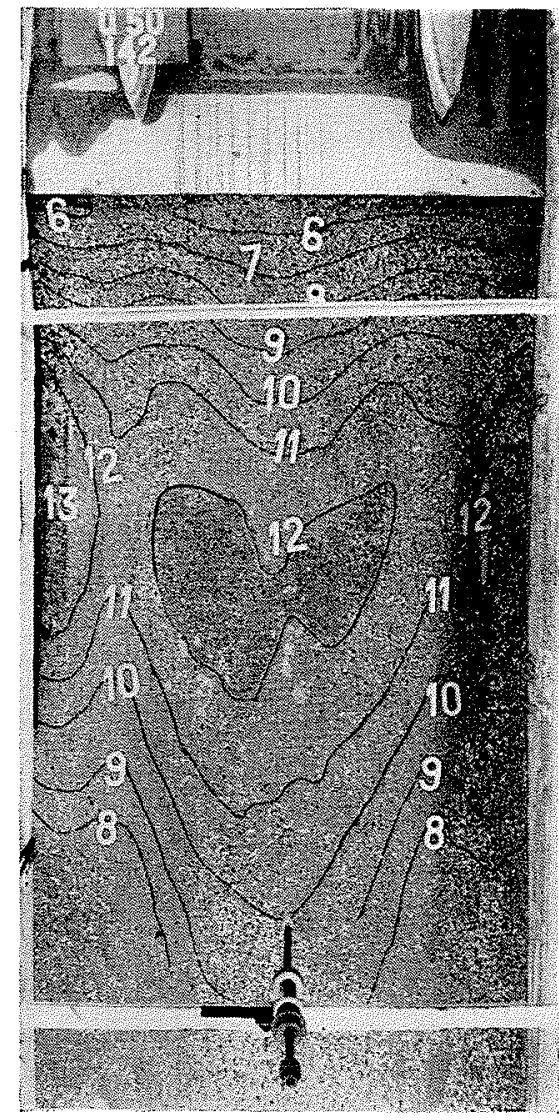

b) piles semi-triangulaires.

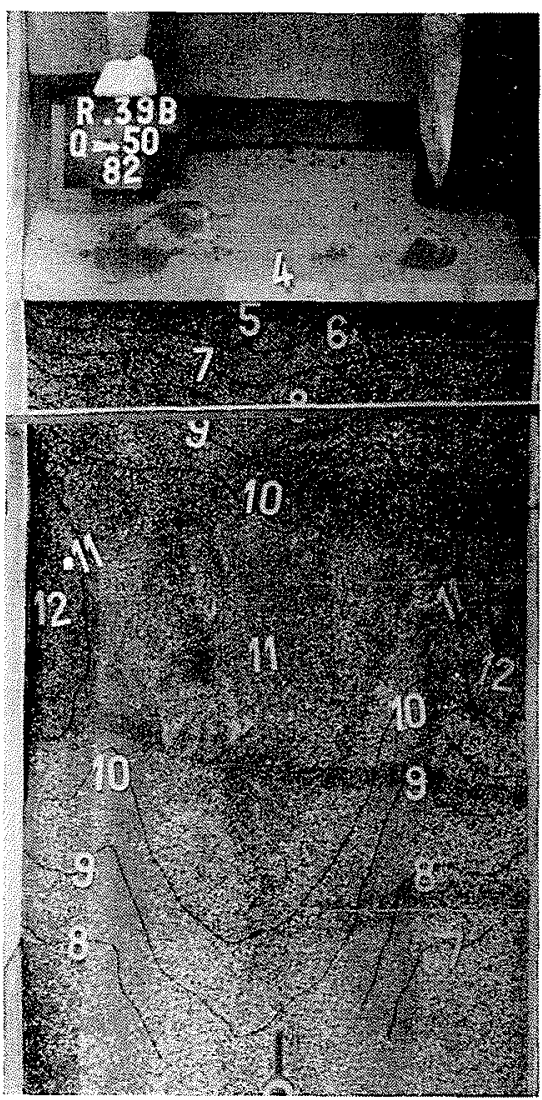

c) piles triangulaires.

Fıc. 12. - Influence de la forme en plan des piles.

Profil $n^{\circ} 39$ sans rugosité.

Reliefs obtenus après l'essai-type de longue durée (T=24h $Q=50 \mathrm{l} / \mathrm{s}$ ).

de cette méthode, de doctrine précise ou du moins solidement justifiée.

On considère intuitivement que, pour constituer un tapis en enrochement, il suffit de choisir un matériau qui ne soit pas entraîné par le courant, donc aussi gros que possible. Or, pour qu'il assure pleinement son rôle, le tapis doit aussi empêcher le matériau sous-jacent d'être luimême entraîné. Cette deuxième condition est contradictoire avec la première. En effet, un tapis constitué par des gros blocs augmente la turbulence des couches profondes de l'écoulement. Les tourbillons créent localement des pressions qui peuvent être très inférieures à la pression hydrostatique régnant dans la couche sousjacente. D'où la naissance d'un courant ascendant qui entraîne les matériaux à travers les vides du tapis. La deuxième condition n'est pas alors assurée. Du fait de l'érosion de son support, le tapis s'abaisse et se désagrège et des affouillements se développent qui peuvent être supérieurs à ceux qui se seraient produits en l'absence du tapis.
C'est à partir de cette considération du courant ascendant que Posey [8], ayant surtout en vue la protection des parements amont des barrages en terre, a proposé un matériau ayant les caractéristiques d'un filtre dit « de Terzaghi Vicksburg » et dont la granulométrie serait définie par les inégalités suivantes, $D_{p}$ se rapportant au filtre et $d_{p}$ au support :

$$
\begin{aligned}
& \frac{\mathrm{D} 15}{d 85}<5 \\
& 4< \frac{\mathrm{D} 15}{d 15}<20 \\
& \frac{\mathrm{D} 50}{d 50}<25
\end{aligned}
$$

Pour y' satisfaire pratiquement, on peut employer des matériaux de carrière, à condition de prendre des précautions à l'extraction et de corriger, par tamisage, la granulométrie du toutvenant. Mais cette solution est évidemment assez onéreuse. 
Nous pensons que pour réaliser un tapis de protection au pied d'un barrage établi sur un lit de gravier, il est possible de trouver un matériau beaucoup plus économique extrait a partir du gravier lui-même. Nous avons été conduits à cette hypothèse par les résultats de l'une des études sur le charriage de la Durance que l'Electricité de France a dû entreprendre à l'occasion du projet d'aménagement de la rivière.

Il s'agit d'une série de prélèvements dans une nasse à gravier dụ matériau charrié par la Durance à différents débits et en différents points de la section de jaugeage du Pont de Mirabeau. Leur analyse granulométrique démontre que le matériau participant au charriage, pour les débits moyens, est nettement plus fin que le matériau prélevé sur les bancs (le $d m$ est deux fois plus petit). Les plus gros éléments du lit de la rivière ne sont pratiquement jamais entrainés. Ils ne le seront pas non plus à l'aval du barrage, à condition évidemment que le mairtien du ressaut sur le radier soit assuré.

D'autre part, dans le cas de lits à granulométrie étendue, il est toujour's possible d'extraire, par tamisage au-dessus d'un certain diamètre, un matériau compatible avec la condition du filtre. Le matériau obtenu, satisfait donc, en définitive, aux deux conditions d'emploi en enrochement et peut servir à la réalisation d'un tapis de protection économique et efficace. L'épaisseur du tapis doit être de plusieurs fois le diamètre maximum, afin que, même s'il ne reste plus en surface que les gros éléments, la couche inférieure intacte soit suffisante pour jouer encore son rôle de filtre.

Pratiquement, la méthode revient à utiliser comme enrochement le rebut des ballastières existant le long de la rivière, ou. installées pour le chantier.

\section{b) RÉSUMÉ DEs ESSAIS :}

Nous avons procédé à quelques essais sur cette question, dont les résultats illustrent les considérations précédentes.

Dans une première série d'essais, nous avons utilisé des cubes de $1 \mathrm{~m}^{3}$ en les disposant d'abord en vrac, puis en ordonnant la couche supérieure suivant un tapis continu, à la cote de l'extrémité du radier. Dans les deux cas, la fosse d'équilibre obtenue a été du même ordre qu'en l'absence d'enrochements. Les blocs s'enfoncent ou finissent par être entraînés, soit dans les parties basses de la fosse, soit même plus à l'aval.

L'étude a été ensuite reprise avec un matériau extrait du matériau de fond par tamisage à un diamètre réel de $200 \mathrm{~mm}$. Une quantité égale à celle des blocs a été répandue sur une longueur de $60 \mathrm{~m}$ à l'aval du seuil en une épaisseur d'en- viron $1 \mathrm{~m}$. En outre, ce répandage n'a été effectué qu'après quelque temps de fonctionnement, c'est-à-dire sur une fosse déjà amorcée. Nous pensons que cette précaution, qui revient à at tendre, par exemple, un an avant de mettre les enrochements, conduit finalement à une réduction très importante des dépenses ultérieures d'entretien.

La prolongation des essais jusqu'à l'équilibre a permis de constater que, par ce procédé, la fosse se trouve figée dans l'état qu'elle présentait lors de la mise en place des enrochements (cf. fig. 13). L'effet de protection a donc été parfaitement atteint à laide de ce tapis constitué par les plus gros éléments du fond naturel

\section{V. - Consignes de manouvre d'un barrage à plusieurs passes.}

Dans le cas d'un barrage à plusieurs passes, si l'on voulait évacuer sans dégât le débit limite $Q_{L}$ à travers une seule passe, on arriverait évidemment à des dispositions prohibitives. C'est pourcuoi nous avons dù supposer jusqu'à maintenant, dans nos raisonnements, que ce débit $\mathrm{Q}_{1}$. se trouvait également réparti entre toutes les passes du barrage. Comme il n'en est jamais ainsi, il est indispensable de recourir en dernier ressort à des consignes de manœuvre pour réduire les affouillements.

Le diagramme I de la figure 14 permet d'établir graphiquement ces consignes. On a supposé un barrage à trois vannes indépendantes, ouvertes successivement pour maintenir la retenue amont à un niveau constant.

Avec la solution mise au point à la suite de l'étude sur modèle et fondée sur l'hypothèse d'une répartition uniforme des débits entre les trois passes, un ressaut ordinaire doit se maintenir sur le radier pour le débit $Q_{\mathrm{L}}$. Dans ces conditions, le niveau aval du ressaut (courbe 1) est sensiblement égal au niveau naturel (courbe 2). En général, pour les débits inférieurs à $Q_{\mathrm{L}}$, le niveau aval du ressaut est alors, comme sur la figure 14, toujours inférieur an niveau naturel.

Si tout le débit était évacué par une seule passe, la courbe de garantie du ressaut serait la courbe 3 (affine de la courbe 2 dans le rapport $1 / 3$ ).

A l'aide de ces trois courbes, on peut arriver, par des considérations simples, à définir la variation admissible du niveau à l'aval de chaque passe et, par suite, le diagramme de manœuvre nécessaire pour suivre une montée de crue (diagramme II de la figure 14).

Pour sa première ouverture, chaque vanne $i$ 


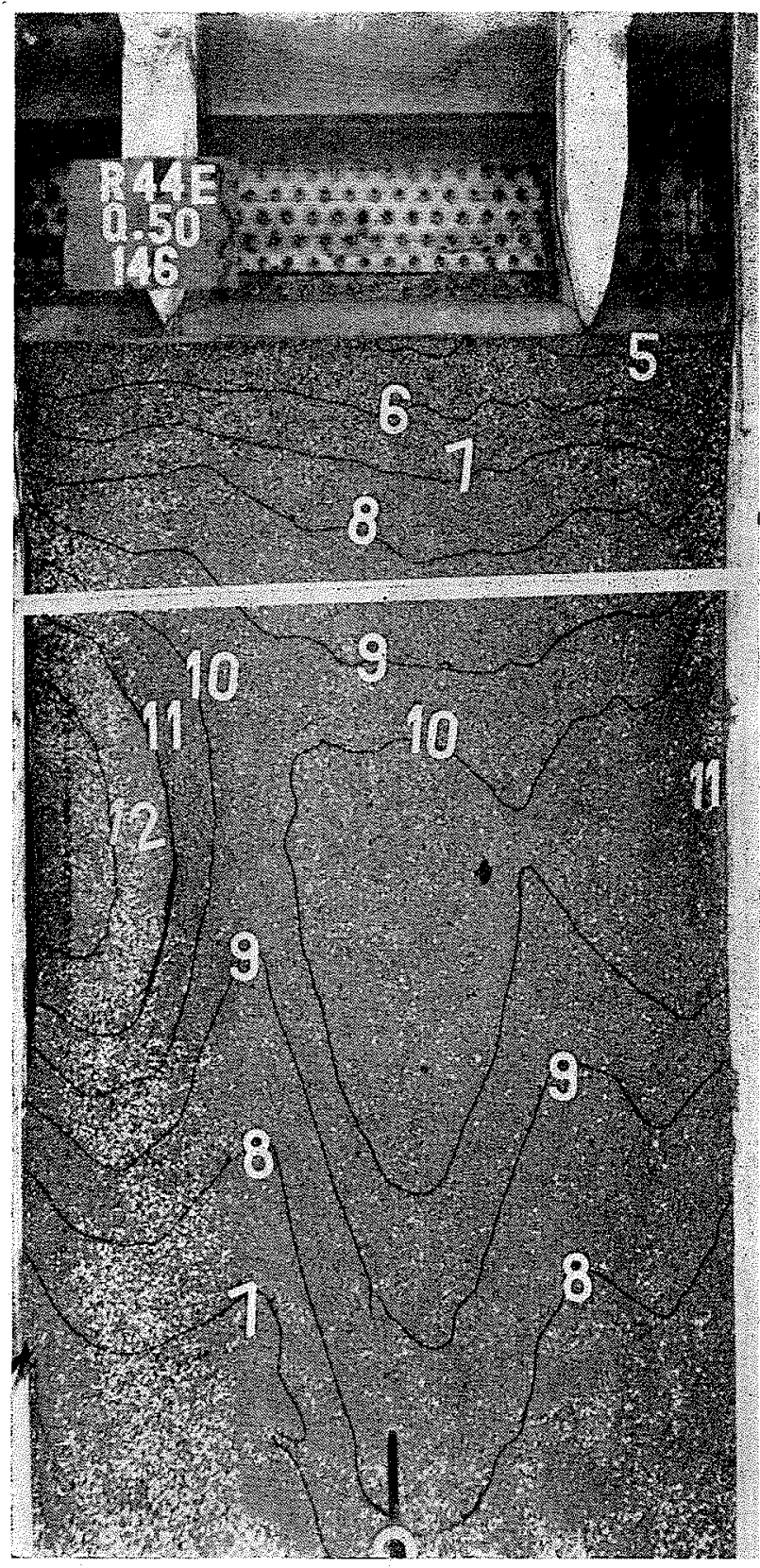

$a$
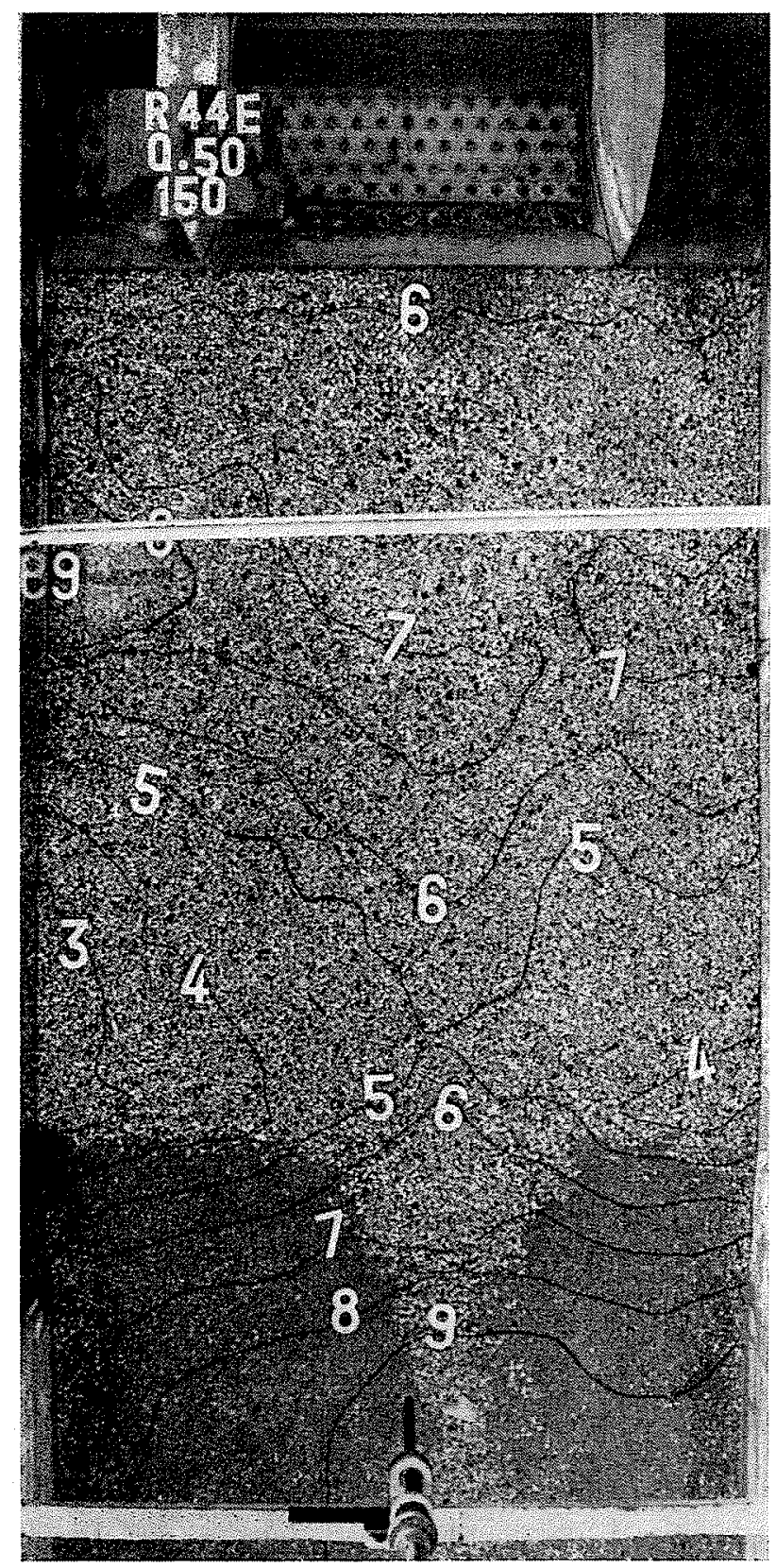

b

Frc. 13. - Effet d'une protection

à l'aide d'enrochements extraits du lit de la rivière, avec la solution adoptée pour le barrage de Cadarache :

— Radier $44 \mathrm{E}$ : profil 39 raccourci de $5 \mathrm{~m}$, rugosité décroissante;

- Piles semi-triangulaires.

Reliefs obtenus après l'essai-type de longue durée $(T=24 \mathrm{~h} \quad Q=50 \mathrm{l} / \mathrm{s})$ :

a) sans enrochement;

b) avec enrochement naturel mis en place après $3 \mathrm{~h}$ d'essai. 
peut évacuer au maximum un débit tolal $\mathrm{Q}_{i}$. Audelà, il faut ouvrir une autre vanne. A partir du débit limite correspondant à la dernière vanne, on
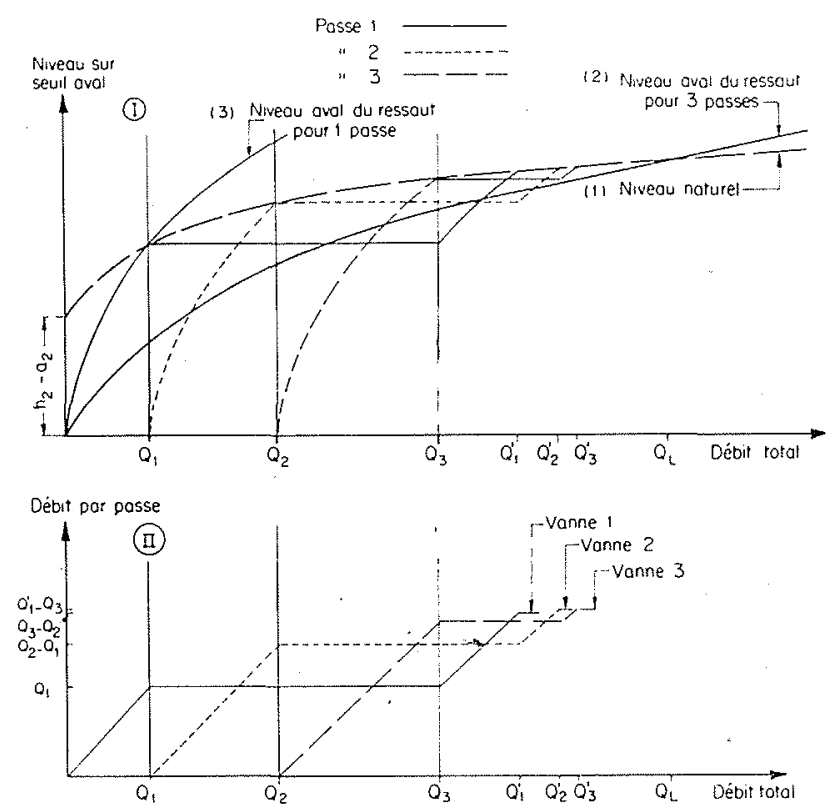

Fig. 14. - Etablissement des consignes de mancuve d'un barlage a trois passes.

peut à nouveau ouvrir la vanne $i$ jusqu'au débit $Q_{i}^{\prime}$, et ainsi de suite.

Une consigne souvent pratiquée consiste à ne jamais admettre un écart de débit, entre vannes, supérieur à $Q_{1}$. Celte règle apparaît trop restrictive pour les débits moyens, mais peut devenir insuffisante pour les crues. Le graphique montre même que, pour les crues voisines de $Q_{\mathrm{L}}$, il faudrait théoriquement en venir à un asservissement total. En fait, l'érosion résultant de ces crues esl limitée par deux raisons: leur très faible fréquence et l'existence d'un charriage qui se produit en général à travers le barrage pour ces débits.

Il est à noter que l'ordre d'ouverture des vannes n'est pas indifférent : il est préférable de commencer par ouvrir la vanne centrale, puis les vamnes voisines en n'utilisant celles qui sont en rive que lorsque l'importance de la crue le rend nécessaire. On réduit ainsi le risque d'affouillements des ouvrages de raccordement aval.

D'autre part, l'asservissement de certaines vannes les unes aux autres serait évidemment toujours souhaitable, puisqu'il réduit la probabilité d'avoir une différence supérieure à une valeur donnée entre le niveau naturel et le niveau nécessaire au maintien du ressaut.

Remarquons, enfin, que d'autres consignes doivent être établies selon la même méthode, en supposant qu'une passe se trouve inutilisable. Dans ce cas, il faut en outre prendre la précaution de réduire le débit à travers les passes voisines à la valeur minimum compatible avec les consignes, cela afin de limiter l'importance des courants de retour dont l'effet est toujours d'accélérer les affouillements.

\section{CONCLUSION}

Parmi les problèmes posés par la construction d'un barrage en rivière, l'un des plus délicats à résoudre est celui de la réduction des affouillements creusés dans le lit aval par les eaux restituées. La méthode qui présente le maximum de garantie, lorsqu'elle est applicable, consiste à dissiper l'énergie de ces eaux par formation d'un ressaut hydraulique sur un ouvrage de réception approprié.

Nous ayons montré que le calcul permet de déterminer la solution la plus simple que l'on puisse imaginer d'un radier horizontal disposé au pied du déversoir. On peut se contenter de ce résultat pour choisir la largeur économique du barrage au cours des études d'avant-projet.

Pour la mise au point définitive, une étude sur modèle reste indispensable. Dans le cas d'un barrage à plusieurs passes, on a fait apparaître quelles précautions il y avait lieu de prendre dans l'interprétation des résultats. On opère, en général, dans un canal où l'on ne représente qu'une seule passe. Or, le procédé ne reproduit pas fidèlement le déchaussement du pied du barrage complet. Ce déchaussement peut en effet atteindre localement la cote extrême de l'ensemble de la fosse obtenue en canal, et cela, par suite d'une inégalité de débit entre les passes, même avec des consignes sévères d'exploitation.

Le mode opératoire revient done à soumettre chaque disposition envisagée à un essai-type et c'est la cote extrême de la fosse que l'on doit considérer comme critère de classement.

L'étude systématique effectuée d'après ces principes, à l'occasion du barrage de Cadarache, nous a conduit aux conclusions suivantes :

$1^{\circ}$ Le profil en long de radier proposé, qui apporte une nette amélioration par rapport au radier horizontal, comprend une courbe de raccordement à un déversoir Creager, prolongée jusqu'à une cote suffisante pour assurer la formation du ressaut jus- 
qu'à des débits très élevés, puis un profil convexe redressant la veine au niveau $d u$ fond naturel. C'est d'ailleurs la forme à laquelle on aboutit en réduisant l'échelle des longueurs d'un profil naturel de fosse.

$2^{\circ}$ On réduit de moitié la profondeur de la fosse en ajoutant sur la surface du radier une rugosité artificielle décroissante de l'amont vers l'aval et présentant à son extrémité une rugosité égale à celle du fond naturel de la rivière.

$3^{\circ}$ La forme en plan des piles a aussi une influence sensible sur les affouillements. A partir de la remontée du radier, l'épaisseur de la pile doit diminuer afin de réaliser un écoulement divergent qui améliore la stabilité du ressaut et en diminue la longueur.

4" Enfin, quelques essais ont démontré qu'il est possible de stabiliser la fosse d'affouillement avec des enrochements dont la dimension correspond à celle des plus gros éléments du matériau naturel. Bien que ce résultat choque à première vue, il apparaît que l'effet protecteur risque d'être compromis si l'on utilise des enrochements homogènes de plus gros diamètre.

L'ensemble de ces conclusions pratiques résulte d'ailleurs logiquement de considérations théoriques simples, dont le principe est de limiter au minimum la discontinuité imposée par le barrage aux conditions naturelles d'écoulement.

Pour terminer, nous avons proposé une méthode graphique pour l'établissement des consignes de mancuvre, sans lesquelles toutes les mesures précédentes risqueraient d'être inopérantes sur un barrage à plusieurs passes.

Du fait de leur généralité, l'ensemble des conclusions de cette étude devrait présenter un certain intérêt pour d'autres ouvrages analogues. Les considérations qui ont été développées et justifiées par les résultats obtenus peuvent réduire les tâtonnements dans ce domaine, encore très empirique, de la limitation des affouillements à l'aval d'un barrage.

\section{BIBLIOGRAPHIE}

1. Smolianofy : Erosion du radier à l'aval d'un déversoir.

Gidrotechniceskoe Stroistel'stoo, février 1952.

2. Erosion à l'aval du barrage de Birsfelden.

Wasserwirtschaft, 41 (1950-1951), Heft 3

3. SCIMEMI : Energia Elettrica, novembre 1939.

4. Schoklitsch : Calcul des fosses d'affouillement it l'aval d'un ouvrage de retenue.

Wasser and Energie Wirtschaft, $n^{\circ}$ 2, février 1951.

5. NaZIR AHMad : Irrigation Research Institute, Pakistan. I.A.H.R., A.S.C.E., Minneapolis, septembre 1953.
6. Ter-Arakelian : Contribution au problème des affouillements à l'aval d'un barrage. Gidrotekhniceskioe Stroistel'stoo, $\mathrm{n}^{\circ} 10,1952$.

7. Flierl : Observations d'un ancien constructeur sur le ressaut.

Wasserwirtschaft, janvier 1954.

8. Posey : Quelques considérations fondamentales sur la protection contre l'érosion. I.A.H.R., A.S.C.E., Minneapolis, septembre 1953.

9. Butcher and Atrinson: The causes and prevention of bed erosion with special reference to the protection of structure controlling rivers and canals.

\section{I S C U S S I O N}

président : M. HUPNER

M. le Président félicite M. Fauconnier de sa très intẻressante communication. Il pose les questions suivantes au conférencier qui répond au fur et à mesure :

\section{Premiere gurstion}

Comment l'instabilite du lit de la Basse Durance peut elle se concilier avec l'existence de la formule $h(Q)$ pour tenir compte de l'abaissement du niveau aval par affoullement?

\section{Réponse de M. Fauconnier :}

Malgré cette instabilité locale du lit de la Durance, on définit statistiquement un profil en long de la rivière

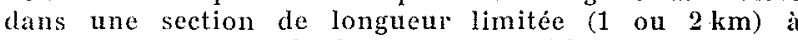
l'amont et à l'aval du barrage : la méthode, qui n'a pu ctre exposée en détail en raison du temps réduit dont disposait le conférencier, se trouve explicitée dans son mémoire (paragraphe $A, 1^{\circ}$ : Données de l'étude. Détermination de la courbe naturelle hauteur-débit).

\section{Deuxième Questron}

M. Escande a signalé jadis que dans un barrage at passes multiples la répartition du débit entre les passes est fonction de l'ordre dans lequel les manouvres d'ouverture se sont succédées : ce phénomène a-t-il été constaté à Cadarache, et les consignes établies pour la mancuvre en tiennent-elles compte?

\section{Réponse de M. Fauconnier :}

Ce phénomène concernant la répartition du débit entre les passes se trouve rapidement masqué dans lo cas d'une rivière à fond mobile par les déplacements de 
l'axe principal d'écoulement à l'amont et par l'évolution de la fosse à l'aval du barrage. Les consignes indiquées sont simplement destinées, d'une part, à maintenir le ressaut sur le radier de facon à réduire au minimum l'énergie d'érosion et, d'autre part, à répartir cetto énergie de façon à réduire au minimum son importance le long des rives.

\section{Troisième Question}

La loi empirique de Flierl, d'après laquelle la longueur du ressaut serait proportionnelle au carré du coefficient de rugosité, est-elle encore valable dans le cas d'un radier extrêmement lisse?

Cette loi très théorique, ne saurait être prise comme formellement exacte : de même que la rugosité n'est pratiquement jamais nulle, il n'y a évidemment pas de ressaut de longueur infinie. Nous ne voulons en retenir qu'une indication de tendance de la liaison existant entre ces deux grandeurs.

En réponse à une question posée par M. le Président, M. Aubert indique qu'il est exact gu'en France, on emploie un peu indifféremment les expressions do "vamne-segment 》 et de 《vanne-secteur 》, alors qu'à l'étranger, et notamment en Allemagne, on ne commet pas cette confusion.

Sans attacher à ce point de pure terminologie une importance exagérée, M. Acbent pense que l'on devrait, comme à l'étranger, appelej barrages à vanne-segment ceux qui comportent seulement une bouchure en forme de segment de cercle (ou plutôt de cylindre), relié à l'axe de rotation par des bras discontinus. Les barrages à vannes-secteur, qui sont habituellement manouvrés par lit chute, comportent au contraire, un plan radial plein et des fermetures triangulaires latérales, lensemblo constituant ainsi un volume et non pas seulement une surface courbe, comme dans le cas des barrages à vannesegment.

\section{Intervention de M. Aubert :}

M. I'Inspecteur général AUBERT fait ici une autre importante intervention, dont il a bien voulu rédiger lui-même le texte ci-après :

Je suis d'accord sur tout ce qu'a dit M. Fauconner et je considère que son travail (que je me propose de relire et d'étudier plus complètement) constitue une contribution importante à la question des affouillements en aval des barrages.

Ainsi qu'il l'a dit, il est très important de donner aux jeunes ingénieurs qui établissent des projets des idées simples auxquelles ils puissent se raccrocher.

A cet égard̃, il n'est peut-être pas inutile de rappeler des vérités presque évidentes.

Les affouillements en aval d'un barrage se produisent du fait de l'énergie à dissiper localement et dont. l'expression est donnée par la formule $10 \mathrm{Oh}$.

A beaucoup de barrages, $O$ est pratiquement nul lorsque $h$ est maximum, tandis que $h$ devient très faible lorsque le barrage est complètement ouvert.

Pour ces barrages, la situation la plus défavorable est celle de l'ouvrage partiellement ouvert. C'est par exemple, le cas du barrage de Kembs sur le Rhin, où les ordres de grandeur peuvent être les suivants : $c=1.000 \mathrm{~m}^{3}$ avec $h=5 \mathrm{~m}$. La puissance $\dot{a}$ dissiper est alors de $50.000 \mathrm{~kW}$ et on conçoit qu'il ait été nécessaire d'établir une puissante protection des berges sur plus de 500 mètres de longueur, ceci indépendamment des dis. positions prises par ailleurs pour protéger le fond.

Dans d'autres cas, le maximum du produit $Q h$ s'obtient Iorsque le barrage est complètement ouvert et je demande à M. Fauconvier s'il en est ainsi de l'ouvrage qu'il a étudié (réponse affirmative).

Dans de telles conditions, la relation qui relie l'énergie à dissiper au débit $Q$ est une fonction du débouché linéaire donné au barrage mobile et de la hauteur don- née à la bouchure ou, ce qui revient au mème, de la surface de la bouchure mobile.

Dans ces conditions, l'étude des affouillements et des protections nécessaires, faite pour une surface de bouchure déterminée, devrait être reprise pour une surfaco de bouchure différente obtenue, par excmple, en conservant les dispositions en plan et en abaissant le seuil sur lequel s'appuie la bouchure mobile. Théoriquement, c'est après un certain nombre de tâtonnements de ce genre que l'on aboutirait à la solution la plus favorable. Il est évident que dans certains cas, le flair de l'Ingénieur permet de réduire, ou même de supprimer, les tâtonnements en question.

En ce qui concerne la dissipation de l'énergie produite par la chute, on peut, pour poser le problème d'unc facon claire, distinguer deux lignes de conduite s'opposant l'une à l'autre, la solution pratique à adopter constituant habituellement un compromis entre ces deux idées extrêmes.

\section{PrEMÈRE MËTHOD}

Elle consisterait à donner aux maçonneries du radier des dispositions telles (par exemple au moyen d'un parafouille aval profond et indestructible) que l'on puisse impunement admettre la production d'affouillements très considérables en aval.

Dans la fosse ainsi produite se développent des tourbillons importants qui produisent la dissipation d'énergie nécessaire, les eaux ayant alor's retrouvé, au point où elles s'écoulent en aval, la disposition normale des vitesses triangulaires (ou trapézö̈dales) comportant de faibles vitesses sur le fond et de plus fortes vitesses en surface.

\section{DEUXǏ̆ME MÉTHODI}

Elle consiste à donner aux maçonneries des dispositions telles, que l'eau s'écoule en aval du radier dans des conditions qui respectent la loi de répartition naturelle des vitesses dont il vient d'etre question.

C'est à cette deuxième méthode que M. Fauconnen s'est tout d'abord attaché et, en vue d'aboutir à cette dissipation d'énergie sul le malier, il a examiné dans quelles conditions le ressaut utilisé par lui comme moyen principal de dissipation pouvait être fixé sur la partie masconnée sans risquer de s'échapper jamais en aval.

Il a d'ailleurs ajouté, et je suis pleinement d'accord avec lui, que si cette fixation du ressaut etait une condition nécessaire, elle ne constituait pas une condition suffisante pour que l'on obtienne une répartition correcte des vitesses.

Il a également étudić comme adjuvant, se rattachant lui aussi à la deuxième méthode une augmentation de la rugosité des maçonneries, obtenue, par exemple, au moyen de saillies. Celles-ci présentent, à mon avis, le danger" d'être dans certains cas (et ce n'est peut-être pas celui de l'onvrage de M. Fauconvier) exposées à une destruction rapide.

M. Faucoviser a finalement ea recours aussi a uno vtilisation partielle de la première méthode, consistant à laisser se produire, en aval des maçonneries, une fosse d'importance limitée, dont la surface est tapissée par des matérianx de dimensions convenables.

Je pense qu'il s'agit là d'une solution tout à fait satisfaisante et que je considère même comme très générale. Il serait habituellement beaucoup trop coutteux de chercher à réaliser des dispositions telles que l'on arrive à eviter complètement la production d'une fosse en aval du radier.

Aux deux procédés de dissipation d'énergie mentionnés par M. Fauconsmse, qui comportent la production d'un ressaut dissipateur ou l'acceptation d'une fosse en aval, je voudrais en ajouter un troisième, auquel on a moins souvent recours et qui n'est d'ailleurs à envisa- 
ger que lorsque le maximum du produit $Q h$ ne correspond pas à l'ouverture totale.

Il consiste à essayer de dissiper l'énergie de la chuto a la traversée mème de la bouchure.

Avec de grands éléments de bouchure, on obtient un resultat assez satisfaisant rest-à-dive la production de chocs entre les masses d'eau ayant des orientations dif. férentes) soit par lame déversante aérée, soit même par lame inférieure très minee. Malheureusement, ces deux procédès deviennent tout à fait inefficaces dès que le débit écoulé est important, e'est-à-dire dès que la situation devient réellement dangereuse.

Les barrages à petits éléments présentent, à cet égard, un avantage.

Je parlerai à peine des barrages à fermettes et à aiguilles, qui sont aujourd'hui justement abandonnées en raison d'autres inconvénients, mais qui permettraient de pratiquer une série de petites ouvertures discontinues, contribuant $\dot{a}$ la formation de nombreux tourbillons amortisseurs.

Les seuls barrages modernes à petits éléments sont les barrages à hausses, et je voudrais dire quelques mots du barrage de Meaux snl la Marne, qui a été construit il y a une vingtaine d'années par M. Rumpler, l'actuel Directeur des Routes au Ministère des Travaux publics, mais qui n'a malheureusement fait l'objet d'aucune publication.

Je précise, tout d'abord, que le barrage de Meaux appartient à la catégorie des ouvrages pour lesquéls ce sont les ouvertures partielles qui donnent lieu à la plus forte dissipation d'énergie, car la chute s'annule lorsque le bariags est complètement ouvert.

Les ingénieurs chargés de l'exploitation de ce barrage ont soin de' maintenir toujours les hausses dans la disposition « en chicanes » (sauf, bien entendu, lorsque le débit est quasi nul et que toutes les hausses sont redressées). Lesdites chicanes sont plus ou moins nombreuses et plus ou moins ouvertes, la chute entre lamont ef l'aval étani alor's de l'ordre de 4 à 5 mètres.

Cette disposition « en chicanes », donnant lieu a la prodiction de courants liquides parfaitement symétriques et directement opposés les nus aux autres, trans- forme l'eau en une émulsion. Celle-ci est huileuse et légèrc et elle s'écoule à la surface du bief aval en lais. sant pratiquement immobile le matelas d'eau directement en contact avec le fond.

Il est intéressant de signaler que, d'après les ingénieurs de Meaux, et bien que le radier en aval des hausses soit parfaitement lisse, les sables et graviers qui constituent le fond de la rivière restent absolument immobiles immédiatement en aval de l'ouvrage. C'est sans doute l'un des très rares ouvrages au monde, construits sur fond affouillable, où l'on ne s'est pas trouvé dans l'obligation de protéger le fond par des enrochements sur une certaine longueur en aval des maçonneries.

Ce procédé de dissipation de l'énergie à la traverséo même de la bouchure est à retenir, car il permet de réaliser des économies importantes sur le radier, qui peut être, toutes choses égales par ailleurs, fortement raccourci.

On peut rappeler, à cet égard, que l'estimation de la dépense d'un ouvrage contenant une partie mobile doit toujours porter sur l'ensemble, l'erreur ayant été quelquefois commise de choisir un type de bouchure supposé économique, sans se préoccuper suffisamment des conséquences qui en résulteraient pour la construction des maçonneries.

\section{M. le Président remercie M. AunErT.}

M. Fauconner signale qu'un essai, qu'il a fait en canal, Iui a suggéré une petite exception à la formule 10 oh donnant l'énergie dissipée en affouillement. Le canal était constitué d'un fond mobile en sable ayant atteint son profil d'équilibre. Au milieu de ce canal, on réalisait sur $1 \mathrm{~m}$ de longueur une chape en ciment se substituant exactement an profil mobile. En poursuivant l'essai dans ces conditions, il se formait à l'aval de la plaque une fosse d'affouillement, décelant la dissipation d'une énergie résultant de la variation locale de la rugosité de fond, alors que la formule donnerait une energic nulle.

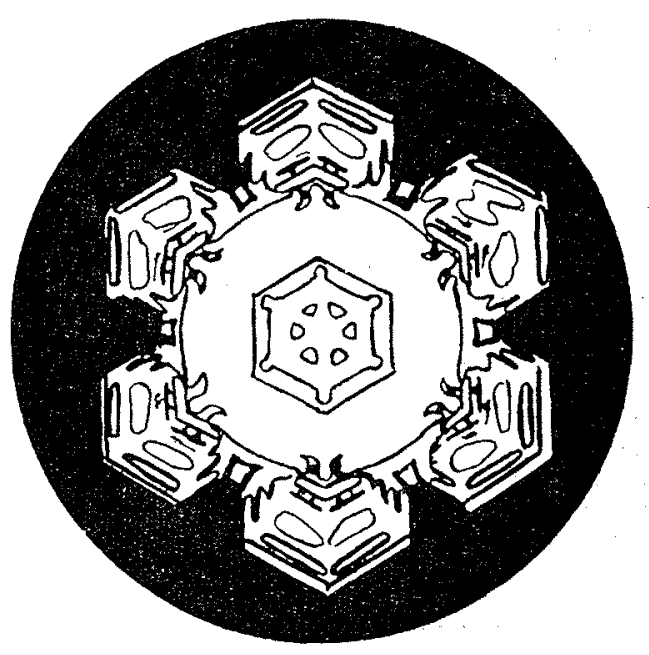

